\title{
RESEARCH
}

Open Access

\section{Wild plants used by the Lhoba people in Douyu Village, characterized by high mountains and valleys, in southeastern Tibet, China}

Wen-Yun Chen $^{1+}$, Tao Yang ${ }^{1,2+}$, Jun Yang ${ }^{1}$, Zhu-Chuan Qiu ${ }^{1,2}$, Xiao-Yong Ding ${ }^{1,2}$, Yu-Hua Wang ${ }^{1}$ and Yue-Hu Wang ${ }^{1 *}$ (iD

\begin{abstract}
Background: Douyu Village, inhabited by the Lhoba people, is situated within the Eastern Himalayas, in southeastern Tibet, China. The village is located among high mountains and valleys, which feature complex terrain with cold and dry climates and distinctive vegetation types and species. The Lhoba people in this village are isolated from other groups in China. The Lhoba people have lived in this village since the 15th century and mainly depended on gathering, hunting, and swidden agriculture before the 1960s. Because they have a long history and live under extreme climatic, geographical, and ecological conditions, the Lhoba people in Douyu Village may have unique traditional knowledge about wild plants. Thus, this research aims to record the traditional botanical knowledge of the Lhoba people in Douyu.

Methods: An ethnobotanical study was conducted on the Lhoba people in Douyu Village in Longzi County, Tibet, China. Semi-structured interviews and group discussions with informed consent were used in the study. We interviewed 41 informants (14 key informants) between 18 and 75 years of age. All information was collected, organized, and compiled into "use reports" for quantitative analysis. The informant consensus factor (ICF) was used to determine the homogeneity of the informants' knowledge of medicinal plants, while the cultural importance index (Cl) was used to estimate the cultural importance of shared species.
\end{abstract}

\footnotetext{
* Correspondence: wangyuehu@mail.kib.ac.cn

'Wen-Yun Chen and Tao Yang contributed equally to this work.

'Key Laboratory of Economic Plants and Biotechnology and the Yunnan Key Laboratory for Wild Plant Resources, Kunming Institute of Botany, Chinese Academy of Sciences, Kunming 650201, People's Republic of China Full list of author information is available at the end of the article
}

(c) The Author(s). 2021 Open Access This article is licensed under a Creative Commons Attribution 4.0 International License, which permits use, sharing, adaptation, distribution and reproduction in any medium or format, as long as you give appropriate credit to the original author(s) and the source, provide a link to the Creative Commons licence, and indicate if changes were made. The images or other third party material in this article are included in the article's Creative Commons licence, unless indicated otherwise in a credit line to the material. If material is not included in the article's Creative Commons licence and your intended use is not permitted by statutory regulation or exceeds the permitted use, you will need to obtain permission directly from the copyright holder. To view a copy of this licence, visit http://creativecommons.org/licenses/by/4.0/. The Creative Commons Public Domain Dedication waiver (http://creativecommons.org/publicdomain/zero/1.0/) applies to the data made available in this article, unless otherwise stated in a credit line to the data. 
Results: A total of 91 wild species ( 90 vascular plants and 1 fungus) belonging to 71 genera and 39 families utilized by the Lhoba people in Douyu were documented. Of these species, Pimpinella xizangense and Wikstroemia lungtzeensis are endemic to Longzi County, while Sinopodophyllum hexandrum and Paeonia ludlowii are endangered species in China. All habitats, from the field vegetation at the valley bottoms to the alpine shrubland and meadows, were used for plant collection, and the numbers of species of plants collected from the various vegetation types (except for fields) decreased with increasing altitude. Our study found that 55 species are edible plants and fungi, 29 species are medicinal plants, and 38 species are used for other purposes. Medicinal plants are used for 11 categories of diseases, among which diseases of blood-forming organs (ICF $=0.96)$ and gastrointestinal diseases (ICF $=0.95)$ exhibited the highest ICF values. Based on the CI values, the most important plants in this study area are Berberis xanthophloea, B. kongboensis, Sinopodophyllum hexandrum, Vicatia thibetica, and Hippophae rhamnoides subsp. gyantsensis. Moreover, a comparison of the wild plants used by Lhoba ethnic groups in three counties in China showed significant differences among these regions.

Conclusions: Our study demonstrates that the wild plants utilized by the Lhoba people in Douyu Village are highly diverse, at 90 plant and one fungal species, which reflects not only the number of species but also their diversified functions. The extreme climatic, geographical, and ecological conditions of Douyu within the high mountains and valleys of the Eastern Himalayas potentially affect the Lhoba people's culture, including plant utilization practices, and contribute to the rich diversity of the wild plants used by the local people.

Keywords: Ethnobotany, Traditional knowledge, Lhoba people, Eastern Himalayas, Semiarid climates, Tibet

\section{Introduction}

The Eastern Himalayas are characterized by complex topographic terrain with different altitudinal ecological zones [1] and feature a diverse array of vegetation types and rich plant diversity [2-5]. This region is also extremely culturally diverse [2, 6-8]. Wild plants not only contribute to the sustenance of the Eastern Himalayan tribal communities but also form an integral part of the culture and traditions of these communities, where a large number of wild plants are used for various purposes, such as for food, medicine, fodder, fuel, dyes, essences, rituals, and other multifarious purposes by local people [9-12]. In particular, in comparison to other indigenous populations, those in high-altitude regions may be more closely dependent on locally available wild plant resources due to uncertainty related to agricultural practices and low agricultural yields at high altitudes [13, 14], and ethnobotanical investigations of these communities could contribute to specialized knowledge about the culture of wild plant utilization by local people in high-altitude areas [11, 13, 15].

Douyu Village, a Lhoba-inhabited area, is located in Longzi County in the Eastern Himalayas, southeastern Tibet, China $[16,17]$. The Lhoba people have lived in Douyu since the 15th century AD. They show substantial cultural diversity based on their long-term existence and cultural integration [17]. Since the 8th century AD, Lhoba ethnic groups have been concentrated in the Lhoyü area in the Eastern Himalayas [18]. In China, the Lhoba people are distributed in Milin County, Medog County, Zayü County, and Longzi County in Tibet [19]. The Lhoba ethnic groups include many different tribes, such as the Bo'gaer, Bengni, Miguba, and Mixingba [16, 17, 19]. The Lhoba nationality is one of the smallest populations in China. Its population was 3682 according to the 2010 Census. The Lhoba people in Douyu Village belong to the Bengni tribe. Before the 1960s, they mainly depended on gathering, hunting activities, and swidden agriculture to maintain their livelihoods. For a long time, they made full use of the wild plant resources in the surrounding mountains to meet their daily needs and formed a rich diversity of traditional knowledge about wild plants [17]. However, to date, there have been no studies on the local utilization of wild plants by the Douyu Lhoba people.

The number of useful species appears to vary with altitude in mountainous regions [20-25]. It seems that around villages and fields, the diversity of useful plant species is higher than levels in other areas [8, 20, 25]. Convenience and cultural value contribute to the presence of settlements in the vicinity of areas favorable for plant collection $[8,20,25]$. Moreover, extreme geographical and ecological conditions not only isolate plant populations [26] and result in culturally unique biodiversity $[6,7,14]$ but also shape traditional uses of wild plants $[9,27]$. Wild plants play an important role in daily life in these regions because they contribute to the sustenance of local people and form the diversity of knowledge and culture of plants utilized by local people in exceptional environments [9-12].

In recent years, research on the traditional utilization of wild plants by Lhoba ethnic groups in the Eastern Himalayas of China has been conducted. Previous studies on the Lhoba in Milin County and Medog County 
have shown that the local people have rich traditional knowledge about the uses of wild plants $[9,11,12]$. There are significant differences in the wild plants used by the Lhoba people in Milin and Medog, partly due to the different climatic environments and vegetation types in the two regions [9]. In contrast to the abovementioned studied areas, Douyu Village in Longzi County features a typical landform of high mountains and valleys with complex terrain and great altitude variation. This area experiences temperate and semiarid montane climates and includes various vegetation types and specific subalpine broadleaf deciduous shrubs [28]. In addition, Douyu is isolated from other regions in China due to its unique geographic environment and rough road conditions. Therefore, it is necessary to study whether the Lhoba people of Douyu, with a long history interacting with their living environment under extreme climatic, geographical, and ecological conditions in an isolated high altitude village, may have developed specialized lifestyles, cultures, and traditional knowledge of wild plants.

In the present study, our primary aim is to record the traditional botanical knowledge of the Lhoba people in Douyu Village of Longzi County characterized by high mountains and valleys in the Eastern Himalayas, southwestern China. Utilizing these recorded data, we investigate the distribution of useful plants along the altitude gradient, assess the most important plants used by the local people, and analyze the differences in plants utilized among the different Lhoba tribes in China. Finally, we also discuss the relationships of the wild plants used by the Lhoba people to their particular living environment.

\section{Methods}

\section{Study site}

Douyu Village $\left(28^{\circ} 24^{\prime} 40^{\prime \prime} \mathrm{N} 92^{\circ} 88^{\prime} 11^{\prime \prime}\right.$ E, Fig. 1) is located in south-central Longzi County, Shannan Prefecture, southeastern Tibet. This village is in the Eastern Himalayas among high mountains and valleys. This area features complex terrain with extreme altitude variations from 5500 to $2800 \mathrm{~m}$. Douyu experiences temperate and semiarid montane climates, the annual temperature is $4.2{ }^{\circ} \mathrm{C}$, the average temperature of the coldest month is $4.6{ }^{\circ} \mathrm{C}$, the average temperature of the hottest month is $13.1{ }^{\circ} \mathrm{C}$, the annual average precipitation is $279.4 \mathrm{~mm}$, and the average humidity is $54.0 \%$. Mountain brown soil and dark brown soil are the major types of soil. The vegetation of the area ranges from subalpine broadleaf

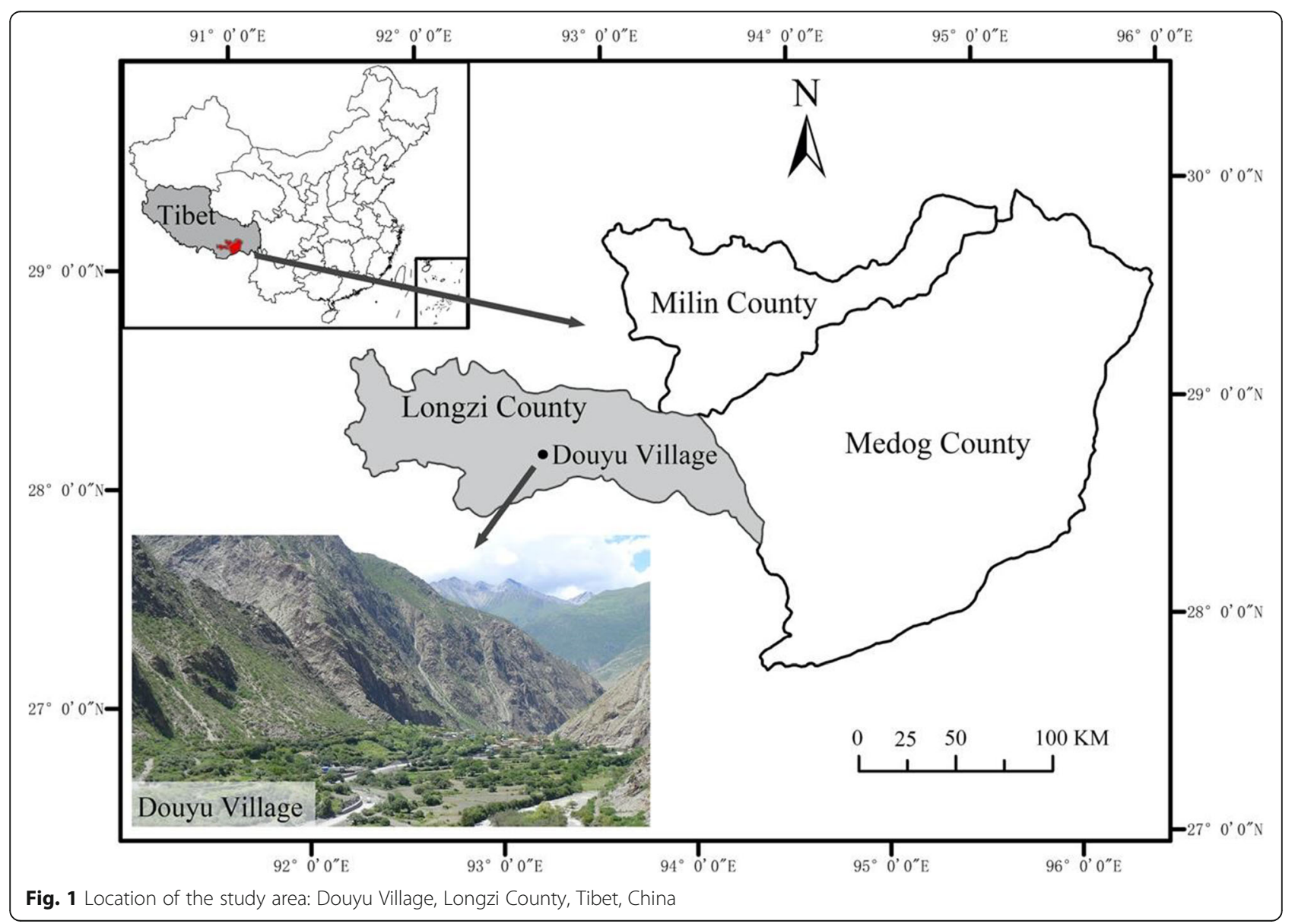


deciduous shrubland at the valley bottoms to alpine shrubland and meadows [28].

The Lhoba ethnic group in Douyu is one of the main groups inhabiting Lhoba. This village is located at the western end of the entire area of distribution of the Lhoba in China. Geographically, the Douyu Village group is isolated from other groups in China, and the Lhoba population in this area only numbers 204. The Lhoba people began to thrive in Douyu in the 15th century [17]. The traditional house is built with timber, and staple foods are highland barley, buckwheat, corn, and potatoes. Barbecuing is the most common food preparation method, and all food can be grilled [17]. Before the 1960s, the Lhoba people mainly depended on gathering, hunting activities, and swidden agriculture to maintain their livelihoods. They have a clear division of labor: men are mainly engaged in hunting and reclaiming farmland, while women are responsible for gathering activities and agricultural production [17]. Their farmland per capita is quite limited, and agricultural yield is low because of extreme living conditions. They have learned to make full use of the wild plant resources in the surrounding mountains to support their daily needs [17]. Moreover, they have traded with Tibetans in San'an Qulin to support this subsistence production system [17, 19]. The Lhoba express faith in the original religion of "all things and spirits". They worship nature, pray for blessings to it, and believe that the Shaman has the power to control spirits of nature $[17,19,29]$. The Lhoba in Douyu belong to the Bengni tribe and use the Bengni dialect, which belongs to the Tani language branch of the Sino-Tibetan language family of TibetoBurman languages. However, the Lhoba people do not have their own written characters [17].

\section{Ethnobotanical surveys and data collection}

Ethnobotanical field surveys were conducted in August 2019 and August 2020. This research was conducted after obtaining permission from the local government and community committee. During the survey, we explained our purpose to the community leaders and requested their assistance, which included providing local guides, translators, and other necessary aid. A total of 41 informants (18 male and 23 female) between 18 and 75 years of age were interviewed. Among them, 14 key informants were selected purposefully and systematically based on the recommendation of the local government, village head, forest rangers, and other personnel, while the other 27 informants were selected from Douyu Village by the snowball method (Table 1). Before each interview, prior informed consent was required, and international ethical standards were observed throughout the research process. After obtaining permission, a key informant interview involving semi-structured
Table 1 Characteristics of informants

\begin{tabular}{lll}
\hline Characteristics & & Number of informants \\
\hline Gender & Male & 18 \\
& Female & 23 \\
Age group & $18-29$ & 11 \\
& $30-39$ & 15 \\
& $40-49$ & 5 \\
& $50-59$ & 6 \\
& $60-69$ & 3 \\
Occupation & $>70$ & 1 \\
& Farmer & 20 \\
& Student & 10 \\
& Government department & 4 \\
& Forest ranger & 2 \\
& Doctor & 2 \\
& Teacher & 2 \\
& Postal worker & 1 \\
& Lhoba & 38 \\
& Tibetan & 3 \\
\hline
\end{tabular}

interviews and group discussions was conducted to collect information about the usage of wild plants and associated traditional knowledge of the local Lhoba people. The major questions were as follows: (1) What plant can be used in your daily life for nutrition, medicine, weaving, or other purposes; (2) what part do you use; (3) why do you use this species; (4) how do you process it; and (5) where and when do you collect it?

The plant specimens were collected in the places of local people's main activities, such as fields, gathering places, and pastures, through transect walks with the key informants and translators. The main sites visited were areas surrounding the village (altitude: 2850-3089 m; vegetation: fields and subalpine broadleaf deciduous shrubland), the valleys behind Douyu Village (3012$3364 \mathrm{~m}$; fields and subalpine broadleaf deciduous shrubland), KuJiuTa (3128-3224 m; subalpine broadleaf deciduous shrubland), DaMu (3351-3695 m; mixed coniferous and broadleaf forests), ZiRong (3384-3695 m; mixed coniferous and broadleaf forests), QiMeiGang (3710-3942 m; mixed coniferous and broadleaf forests, alpine shrubland and meadows), and KaLa Mountain (4286-4762 m; alpine shrubland and meadows).

All plant specimens were identified by referencing the Flora of China. All specimens were collected and deposited at the herbarium of the Kunming Institute of Botany.

\section{Data analysis}

Two ethnobotanical quantitative indices, the informant consensus factor (ICF) and cultural importance index 
(CI), were adopted in this study. The ICF evaluates the distribution of medicinal plant information among information providers and determines the homogeneity of the information providers' knowledge of medicinal plants [30]. The CI is used to indicate the scope of use of each species and determine the diversity of use [31].

The ICF was calculated as follows:

$$
I C F=\frac{N u r-N t}{N u r-1}
$$

where $N_{\text {ur }}$ is the number of user reports of plants used for a certain type of disease. $N_{\mathrm{t}}$ is the number of species reported being used for the specific disease category by informants who mention all the species. The ICF values range between 0 and 1 , and a higher ICF value (close to 1) indicates greater consensus that a species can be used to treat a disease; in contrast, an ICF close to 0 indicates disagreement among the informants [30].

The CI was calculated as follows:

$$
C I_{s}=\sum_{u=u_{1}}^{u_{N C}} \sum_{i=i_{1}}^{i_{N}} \frac{U R_{u i}}{N}
$$

where $N$ is the total number of reports, $N C$ is the total number of plant categories used, and $i$ and $u$ represent the interviewee and the categories of plants used, respectively. CIs is the sum of the proportions of the total numbers of reports for each use category of a given species $(s)$. A higher CI value shows that there are a greater number of different uses of a species [31].
To investigate the number of useful plants within the vegetation zones and habitats across the study area, the collection sites of the species were classified as fields (2800-3100 m), subalpine broadleaf deciduous shrubland (3000-3350 m), mixed coniferous and broadleaf forests $(3350-3800 \mathrm{~m})$, and alpine shrubland and meadows (3800-4700 m) (Fig. 2).

\section{Results}

\section{Diversity of wild plants and their habitats}

This study documented 91 wild species (90 vascular plants and 1 fungus) belonging to 71 genera and 39 families based on the ethnobotanical information on use by the Lhoba people in Douyu Village (Table 2). The majority of plants belonged to Rosaceae (8 species), Compositae (6 species), Fabaceae (5 species), Labiatae (5 species), Liliaceae (5 species), Polygonaceae (5 species), and Ranunculaceae (4 species), and 17 families included only one species. At the genus level, the highest number of species were in the genus Allium (4 species), followed by Cynanchum (3 species) and Polygonum (3 species). Of these plant species, Pimpinella xizangense Shan \& Pu and Wikstroemia lungtzeensis S. C. Huang are endemic to Longzi County [32]. Of the species with restricted ranges, Berberis kongboensis Ahrendt, B. xanthophloea Ahrendt, Berchemia longipedicellata Y. L. Chen \& P. K. Chou, Hedysarum longigynophorum $\mathrm{Ni}$, and Rosa sericea Lindl. f. glandulosa $\mathrm{Yu} \& \mathrm{Ku}$ are endemic to Tibet and are only distributed in the southeastern Himalayas [32]. In addition, Sinopodophyllum hexandrum (Royle) Ying was classified as an endangered species (grade 3) in the
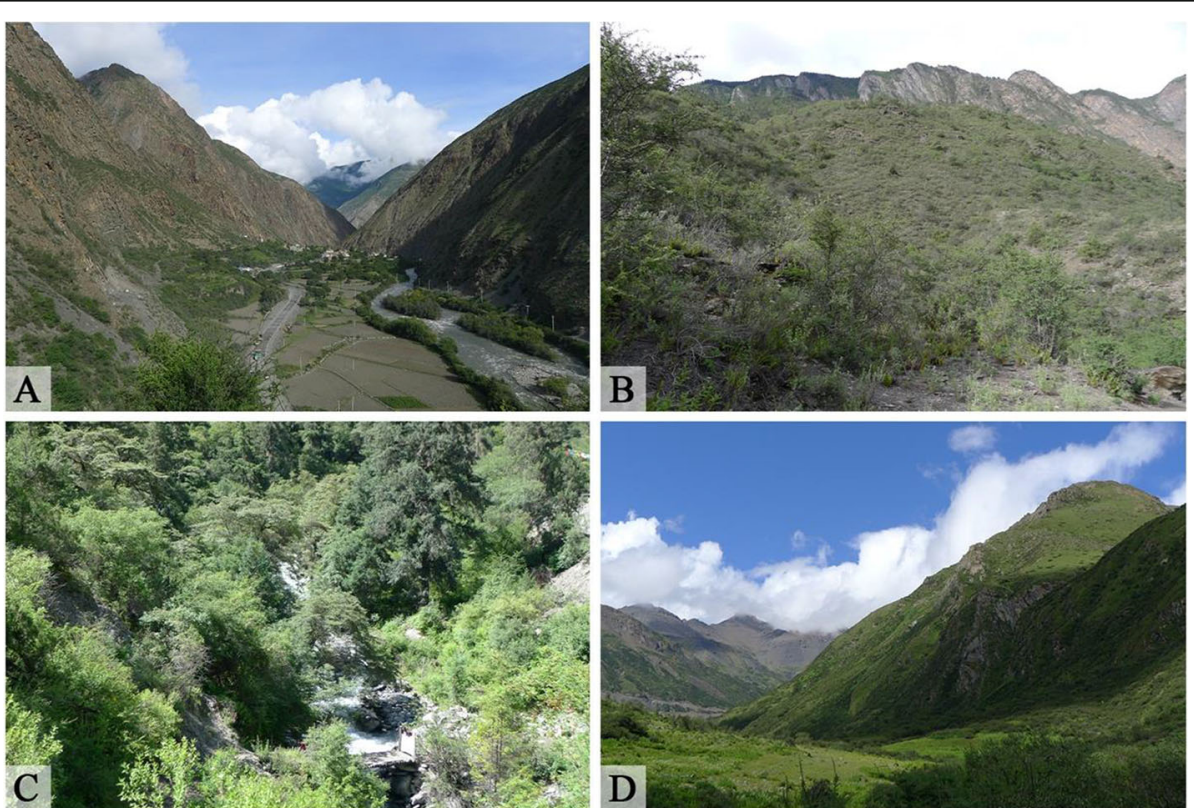

Fig. 2 Plant collection sites. A Fields. B Subalpine broadleaf deciduous shrubland. C Mixed coniferous and broadleaf forests. D Alpine shrubland and meadows 
Table 2 List of plants and fungi used by the Lhoba people in Douyu Village, Longzi County, Tibet, China

\begin{tabular}{|c|c|c|c|c|c|}
\hline Family name & Scientific name & $\begin{array}{l}\text { Local } \\
\text { name(s) }\end{array}$ & $\begin{array}{l}\text { Voucher } \\
\text { number }\end{array}$ & Habit & Habita \\
\hline \multicolumn{6}{|l|}{ Vascular plants } \\
\hline Aceraceae & Acer caudatum Wall. & $\begin{array}{l}\text { long de bi } \\
\text { ya }\end{array}$ & $\begin{array}{l}\text { QTP-EBT- } \\
0245\end{array}$ & Tree & MCB \\
\hline Aceraceae & Acer sp. & $\begin{array}{l}\text { long de bi } \\
\text { ya }\end{array}$ & $\begin{array}{l}\text { QTP-EBT- } \\
0098\end{array}$ & Tree & MCB \\
\hline Araceae & $\begin{array}{l}\text { Arisaema flavum (Forsk.) } \\
\text { Schott }\end{array}$ & $\begin{array}{l}\text { luo ya, duo } \\
\text { ya }\end{array}$ & $\begin{array}{l}\text { QTP-EBT- } \\
0014\end{array}$ & Herb & SBS \\
\hline Asclepiadaceae & $\begin{array}{l}\text { Cynanchum auriculatum } \\
\text { Royle ex Wight }\end{array}$ & da guo & $\begin{array}{l}\text { QTP-EBT- } \\
0078\end{array}$ & Liana & SBS \\
\hline Asclepiadaceae & Cynanchum forrestii Schltr. & $\begin{array}{l}\text { da ge cuo } \\
\text { cuo }\end{array}$ & $\begin{array}{l}\text { QTP-EBT- } \\
0082\end{array}$ & Herb & SBS \\
\hline Asclepiadaceae & $\begin{array}{l}\text { Cynanchum saccatum W. T. } \\
\text { Wang ex Tsiang \& P. T. Li }\end{array}$ & da li & $\begin{array}{l}\text { QTP-EBT- } \\
0027\end{array}$ & Liana & SBS \\
\hline Balsaminaceae & Impatiens cristata Wall. & $\begin{array}{l}\text { duo da, duo } \\
\text { ge le bu }\end{array}$ & $\begin{array}{l}\text { QTP-EBT- } \\
0024\end{array}$ & Herb & SBS \\
\hline Balsaminaceae & Impatiens sp. & zhuo tai ya & $\begin{array}{l}\text { QTP-EBT- } \\
0244\end{array}$ & Herb & ASM \\
\hline Berberidaceae & $\begin{array}{l}\text { Berberis kongboensis } \\
\text { Ahrendt }\end{array}$ & $\begin{array}{l}\text { sha ma de } \\
\text { wei, xia ma }\end{array}$ & $\begin{array}{l}\text { QTP-EBT- } \\
0059\end{array}$ & Shrub & MCB \\
\hline
\end{tabular}

Berberidaceae

Berberis xanthophloea

Ahrendt

Berberidaceae

Sinopodophyllum hexandrum (Royle) Ying

Bignoniaceae

Incarvillea beresowskii Batalin guo duo, xi QTP-EBT- Herb MCB bi guo duo 0037

ke

cuo

(Tibetan)

dang jie men duo

deng jie mai

li, deng ga

ya mi (Tibetan) 0049

xing bu ke

QTP-E

dou yi 0007

QTP-EBT- Shrub SBS 0010

QTP-EBT- Herb ASM 0224

QTP-EBT- Liana SBS 0045

QTP-EBT- Herb ASM 0087

QTP-EBT- Herb Fi 001

P-EBT- Shrub SBS P-EBT- Herb Fi 0100

yong ma ke, QTP-EBT- Herb MCB yong ma 0052
Campanulaceae

Codonopsis convolvulacea ji lu

Kurz. var. vinciflora (Kom.) L.

T. Shen

Campanulaceae Cyananthus incanus Hook. f. \& Thoms.

Caprifoliaceae Sambucus adnata Wall.

Caryophyllaceae Silene viscidula Franch.

Celastraceae

Euonymus tibeticus W. W. Smith

Chenopodiaceae

Chenopodium album L.

su gei, su gu QTP-EBT- Herb MCB

Chenopodiaceae Che

Compositae

ji wo ju wo QTP-EBT- Herb Fi
Anaphalis margaritacea $(\mathrm{L})$ Benth. \& Hook. f.
0.66 Food: beverage, tea substitute, and leaves soaked in water (leaves).

0.66 Food: beverage, tea substitute, and leaves soaked in water (leaves).

1.46 Food: cooked vegetable, used to make soup (leaves).Medicinal: decoction, used to treat high blood pressure (tuber).

0.49 Food: substitute for staple foods (fruits).

0.49 Food: substitute for staple foods (fruits).

1.12 Food: staple food (fruits).Medicinal: decoction, used to treat lumbago, nephropathy, and arthralgia (roots).

1.12 Dye: used to dye wood products yellow (flowers).Food: substitute for staple foods (fruits).

0.24 Artware: seed oil, used to polish furniture (seeds).

2.37 Dye: used for children's face decoration (fruits).Food: vegetable used to make salad (flowers); fruit (fruits); beverage, dry fruit in boiled water to drink (fruits).Medicinal: raw, used to treat diarrhea and stomachache (bark, fruits, and flowers).

2.37 Dye: used for children's face decoration (fruits).Food: vegetable used to make salad (flowers); fruit (fruits); beverage, dry fruit in boiled water to drink (fruits).Medicinal: raw, used to treat diarrhea and stomachache (bark, fruits, flowers).

2.24 Food: fruit (fruits).Medicinal: raw, used to treat anemia and used as a cosmetic to maintain beauty and youth (fruits).Veterinary: decoction or raw, used as an oxytocic for yaks (fruits).

0.12 Food: staple food, ground into powder to make tsampa (seeds).

0.54 Food: substitute for staple food (roots).

0.29 Medicinal: decoction, used to treat colds (whole plants).

1.27 Food: fruit (fruits).Medicinal: decoction, placed on cuts or broken bones to reduce swelling and hasten healing and used to treat anemia (fruits).

0.37 Other: detergent substitute (roots).

0.29 Food: fruit (fruits)

0.88 Food: cooked vegetable, used to make soup (stems and leaves).Medicinal: cooked, used to treat high blood pressure (stems and leaves).

0.68 Food: cooked vegetable (stems and leaves).

0.41 Fuel: kindling (whole plants). 
Table 2 List of plants and fungi used by the Lhoba people in Douyu Village, Longzi County, Tibet, China (Continued)

\begin{tabular}{|c|c|c|c|c|c|c|c|}
\hline Family name & Scientific name & $\begin{array}{l}\text { Local } \\
\text { name(s) }\end{array}$ & $\begin{array}{l}\text { Voucher } \\
\text { number }\end{array}$ & Habit & Habitat $^{\mathrm{a}}$ & $\mathrm{Cl}$ & Local uses (parts used) \\
\hline Compositae & Arctium lappa $\mathrm{L}$. & $\begin{array}{l}\text { a bu xiong } \\
\text { dei, de jiang } \\
\text { ye liang }\end{array}$ & $\begin{array}{l}\text { QTP-EBT- } \\
0084\end{array}$ & Herb & $\mathrm{Fi}$ & 0.88 & Other: used by children to play games (fruits). \\
\hline Compositae & Picris hieracioides $\mathrm{L}$. & $\begin{array}{l}\text { jia luo bing } \\
\text { bing }\end{array}$ & $\begin{array}{l}\text { QTP-EBT- } \\
0012\end{array}$ & Herb & $\mathrm{Fi}$ & 0.32 & $\begin{array}{l}\text { Food: cooked vegetable, used to make soup } \\
\text { (stems and leaves). }\end{array}$ \\
\hline Compositae & $\begin{array}{l}\text { Siegesbeckia pubescens } \\
\text { Makino }\end{array}$ & $\begin{array}{l}\text { ju yi cai, da } \\
\text { bi da mi, ye } \\
\text { gang de } \\
\text { lang }\end{array}$ & $\begin{array}{l}\text { QTP-EBT- } \\
0030\end{array}$ & Herb & $\mathrm{Fi}$ & 0.10 & Medicinal: poisonous plant (whole plants). \\
\hline Compositae & $\begin{array}{l}\text { Taraxacum calanthodium } \\
\text { Dahlst. }\end{array}$ & $\begin{array}{l}\text { kun ma } \\
\text { (Tibetan) }\end{array}$ & $\begin{array}{l}\text { QTP-EBT- } \\
0069\end{array}$ & Herb & SBS & 1.20 & $\begin{array}{l}\text { Food: cooked vegetable, used to make soup } \\
\text { (whole plants).Medicinal: decoction or cooked, } \\
\text { used to treat tumors and gynecologic diseases } \\
\text { (whole plants). }\end{array}$ \\
\hline Compositae & $\begin{array}{l}\text { Taraxacum maurocarpum } \\
\text { Dahlst. }\end{array}$ & $\begin{array}{l}\text { kun ma } \\
\text { (Tibetan) }\end{array}$ & $\begin{array}{l}\text { QTP-EBT- } \\
0009\end{array}$ & Herb & $\mathrm{Fi}$ & 1.22 & $\begin{array}{l}\text { Food: cooked vegetable, used to make soup } \\
\text { (whole plants).Medicinal: decoction or cooked, } \\
\text { used to treat gynecologic diseases (whole plants). }\end{array}$ \\
\hline Crassulaceae & $\begin{array}{l}\text { Sinocrassula densirosulata } \\
\text { (Praeg.) Berger }\end{array}$ & $\begin{array}{l}\text { xia jin xi wo } \\
\text { bing de } \\
\text { gang }\end{array}$ & $\begin{array}{l}\text { QTP-EBT- } \\
0218\end{array}$ & Herb & SBS & 0.27 & $\begin{array}{l}\text { Hunting plant: used as food for lizards and then } \\
\text { local people can collect lizard eggs (whole plants). }\end{array}$ \\
\hline Crassulaceae & $\begin{array}{l}\text { Rhodiola bupleuroides (Wall. } \\
\text { ex Hook. f. \& Thoms.) S. H. } \\
\text { Fu }\end{array}$ & $\begin{array}{l}\text { xia jin xi wo } \\
\text { bing de } \\
\text { gang }\end{array}$ & $\begin{array}{l}\text { QTP-EBT- } \\
0061\end{array}$ & Herb & SBS & 0.27 & $\begin{array}{l}\text { Hunting plant: used as food for lizards and then } \\
\text { local people can collect lizard eggs (whole plants). }\end{array}$ \\
\hline Crassulaceae & $\begin{array}{l}\text { Rhodiola quadrifida (Pall.) } \\
\text { Fisch. et. Mey. }\end{array}$ & $\begin{array}{l}\text { xia jin xi wo } \\
\text { bing de } \\
\text { gang }\end{array}$ & $\begin{array}{l}\text { QTP-EBT- } \\
0015\end{array}$ & Herb & SBS & 0.64 & $\begin{array}{l}\text { Hunting plant: used as food for lizards and then } \\
\text { local people can collect lizard eggs (whole plants). }\end{array}$ \\
\hline Cupressaceae & $\begin{array}{l}\text { Sabina convallium (Rehd. \& } \\
\text { Wils.) Cheng \& W. T. Wang }\end{array}$ & $\begin{array}{l}\text { su gei, su } \\
\text { gei ai xing }\end{array}$ & $\begin{array}{l}\text { QTP-EBT- } \\
0058\end{array}$ & Shrub & SBS & 1.78 & $\begin{array}{l}\text { Food: cooked vegetable, mixed with fruits and } \\
\text { peas to make a salad (fruits).Fuel: fuelwood and } \\
\text { kindling (stems, leaves).Ritual plant: incense } \\
\text { (stems, leaves). }\end{array}$ \\
\hline Elaeagnaceae & $\begin{array}{l}\text { Hippophae rhamnoides L. } \\
\text { subsp. gyantsensis Rousi }\end{array}$ & $\begin{array}{l}\text { la xiong a xi, } \\
\text { la xiong }\end{array}$ & $\begin{array}{l}\text { QTP-EBT- } \\
0021\end{array}$ & Tree & MCB & 2.02 & $\begin{array}{l}\text { Food: fruit (fruits); beverage, fresh or dried fruits } \\
\text { soaked in wine or water (fruits).Medicinal: to } \\
\text { prepare a medical liquor, used to relieve altitude } \\
\text { sickness (fruits). }\end{array}$ \\
\hline Ericaceae & $\begin{array}{l}\text { Rhododendron anthopogon } \\
\text { D. Don }\end{array}$ & la qi la ni & $\begin{array}{l}\text { QTP-EBT- } \\
0090\end{array}$ & Shrub & ASM & 0.73 & Ritual plant: incense (stems, leaves). \\
\hline Ericaceae & $\begin{array}{l}\text { Rhododendron lepidotum } \\
\text { Wall. ex G. Don }\end{array}$ & la qi la ni & $\begin{array}{l}\text { QTP-EBT- } \\
0089\end{array}$ & Shrub & ASM & 0.73 & Ritual plant: incense (stems, leaves). \\
\hline Fabaceae & $\begin{array}{l}\text { Hedysarum } \\
\text { longigynophorum } \mathrm{Ni}\end{array}$ & $\begin{array}{l}\text { zhi ba, ye lu } \\
\text { da bi }\end{array}$ & $\begin{array}{l}\text { QTP-EBT- } \\
0064\end{array}$ & Shrub & SBS & 1.44 & $\begin{array}{l}\text { Agricultural tool: to make rope (roots).Food: } \\
\text { substitute for staple foods (roots).Hunting plant: } \\
\text { firelock fuse (roots). }\end{array}$ \\
\hline Fabaceae & $\begin{array}{l}\text { Indigofera hebepetala Benth. } \\
\text { ex Baker var. glabra Ali }\end{array}$ & bi li mi ni & $\begin{array}{l}\text { QTP-EBT- } \\
0050\end{array}$ & Herb & MCB & 0.20 & Other: detergent substitute (roots). \\
\hline Fabaceae & Medicago sativa $\mathrm{L}$. & de bi & $\begin{array}{l}\text { QTP-EBT- } \\
0101\end{array}$ & Herb & $\mathrm{Fi}$ & 0.80 & Fodder: yak feed (whole plants). \\
\hline Fabaceae & $\begin{array}{l}\text { Piptanthus nepalensis } \\
\text { (Hook.) D. Don }\end{array}$ & $\begin{array}{l}\text { xi wo dong } \\
\text { da bi }\end{array}$ & $\begin{array}{l}\text { QTP-EBT- } \\
0051\end{array}$ & Shrub & $M C B$ & 0.05 & Medicinal: poisonous plant (whole plants). \\
\hline Fabaceae & Vicia cracca L. & $\begin{array}{l}\text { de bi, ye lu } \\
\text { de bi }\end{array}$ & $\begin{array}{l}\text { QTP-EBT- } \\
0085\end{array}$ & Liana & $\mathrm{Fi}$ & 0.71 & Fodder: yak feed (whole plants). \\
\hline Fagaceae & $\begin{array}{l}\text { Quercus aquifolioides Rehd. } \\
\text { \& Wils. }\end{array}$ & ge rui xi & $\begin{array}{l}\text { QTP-EBT- } \\
0254\end{array}$ & Tree & MCB & 0.56 & Ritual plant: sacrificial ceremony (stems, leaves). \\
\hline Gentianaceae & $\begin{array}{l}\text { Gentiana tibetica King ex } \\
\text { Hook. f. }\end{array}$ & $\begin{array}{l}\text { ji xiong } \\
\text { (Tibetan) }\end{array}$ & $\begin{array}{l}\text { QTP-EBT- } \\
0017\end{array}$ & Herb & SBS & 0.24 & $\begin{array}{l}\text { Medicinal: decoction, used to treat high-altitude } \\
\text { pulmonary edema (whole plants); poultice of leaf } \\
\text { epidermis, externally applied to treat scalds and } \\
\text { wounds (leaves). }\end{array}$ \\
\hline Gentianaceae & Swertia hookeri C. B. Clarke & $\begin{array}{l}\text { ren ji, reng } \\
\text { ge }\end{array}$ & $\begin{array}{l}\text { QTP-EBT- } \\
0096\end{array}$ & Herb & ASM & 1.05 & $\begin{array}{l}\text { Medicinal: poultice of crushed roots, used to treat } \\
\text { traumatic injury and wounds (roots); decoction, } \\
\text { used to treat high blood pressure (roots). }\end{array}$ \\
\hline
\end{tabular}


Table 2 List of plants and fungi used by the Lhoba people in Douyu Village, Longzi County, Tibet, China (Continued)

\begin{tabular}{|c|c|c|c|c|c|c|c|}
\hline Family name & Scientific name & $\begin{array}{l}\text { Local } \\
\text { name(s) }\end{array}$ & $\begin{array}{l}\text { Voucher } \\
\text { number }\end{array}$ & Habit & Habitat $^{\mathrm{a}}$ & $\mathrm{Cl}$ & Local uses (parts used) $^{\mathbf{b}}$ \\
\hline Labiatae & $\begin{array}{l}\text { Dracocephalum tanguticum } \\
\text { Maxim. }\end{array}$ & $\begin{array}{l}\text { bi yang gu } \\
\text { lu, zhi yang } \\
\text { guo luo }\end{array}$ & $\begin{array}{l}\text { QTP-EBT- } \\
0065\end{array}$ & Herb & SBS & 1.27 & $\begin{array}{l}\text { Food: cooked vegetable, used to make soup } \\
\text { (stems); seasoning (whole plants). }\end{array}$ \\
\hline Labiatae & $\begin{array}{l}\text { Elsholtzia fruticosa (D. Don) } \\
\text { Rehd. }\end{array}$ & $\begin{array}{l}\text { beng jie de } \\
\text { nang }\end{array}$ & $\begin{array}{l}\text { QTP-EBT- } \\
0046\end{array}$ & Shrub & MCB & 0.39 & Food: seasoning (seeds). \\
\hline Labiatae & Nepeta angustifolia C. Y. Wu & $\begin{array}{l}\text { jiu, da pi de } \\
\text { de ke, de fei } \\
\text { dei dei ke }\end{array}$ & $\begin{array}{l}\text { QTP-EBT- } \\
0066\end{array}$ & Herb & SBS & 0.17 & $\begin{array}{l}\text { Medicinal: veterinary, decoction, used to control } \\
\text { animal ectoparasites (whole plants). }\end{array}$ \\
\hline Labiatae & Phlomis betonicoides Diels & ge ma ma & $\begin{array}{l}\text { QTP-EBT- } \\
0016\end{array}$ & Herb & SBS & 0.56 & Food: cooked vegetable, raw (roots). \\
\hline Labiatae & Salvia przewalskii Maxim. & $\begin{array}{l}\text { hei me bu } \\
\text { yong xie, ge } \\
\text { ma ma }\end{array}$ & $\begin{array}{l}\text { QTP-EBT- } \\
0013\end{array}$ & Herb & SBS & 0.76 & Food: nectar used as seasoning, (flowers). \\
\hline Liliaceae & Allium chrysanthum Regel & $\begin{array}{l}\text { gu di, gu chi } \\
\text { (Tibetan) }\end{array}$ & $\begin{array}{l}\text { QTP-EBT- } \\
0203\end{array}$ & Herb & SBS & 0.93 & Food: seasoning (whole plants). \\
\hline Liliaceae & Allium fasciculatum Rendle & $\begin{array}{l}\text { gu di, lin, da } \\
\text { la bu }\end{array}$ & $\begin{array}{l}\text { QTP-EBT- } \\
0095\end{array}$ & Herb & ASM & 1.00 & Food: seasoning (whole plants). \\
\hline Liliaceae & Allium hookeri Thwaites & da la pu & $\begin{array}{l}\text { QTP-EBT- } \\
0200\end{array}$ & Herb & SBS & 0.66 & Food: seasoning (whole plants). \\
\hline Liliaceae & Allium sikkimense Baker & $\begin{array}{l}\text { ri guo, da la } \\
\text { pu }\end{array}$ & $\begin{array}{l}\text { QTP-EBT- } \\
0092\end{array}$ & Herb & ASM & 0.80 & Food: seasoning (whole plants). \\
\hline Liliaceae & $\begin{array}{l}\text { Polygonatum verticillatum } \\
\text { (L.) All. }\end{array}$ & $\begin{array}{l}\text { rang bu } \\
\text { jiang jiang } \\
\text { (Tibetan) }\end{array}$ & $\begin{array}{l}\text { QTP-EBT- } \\
0018\end{array}$ & Herb & SBS & 1.68 & $\begin{array}{l}\text { Fodder: cooked to feed yaks (whole plants).Food: } \\
\text { cooked vegetable, used to make soup (stems and } \\
\text { leaves); staple food, stewed with potatoes or meat } \\
\text { (rhizomes).Medicinal: decoction, used to treat } \\
\text { nephropathy (whole plants). }\end{array}$ \\
\hline Malvaceae & Malva verticillata $\mathrm{L}$. & jiang ba & $\begin{array}{l}\text { QTP-EBT- } \\
0097\end{array}$ & Herb & $\mathrm{Fi}$ & 0.93 & $\begin{array}{l}\text { Food: cooked vegetable, used to make soup } \\
\text { (stems).Medicinal: cooked, used to treat high- } \\
\text { altitude pulmonary edema (whole plants). }\end{array}$ \\
\hline Pinaceae & $\begin{array}{l}\text { Picea likiangensis (Franch.) } \\
\text { Pritz. var. lirzhiensis Cheng } \\
\text { et L. K. Fu }\end{array}$ & $\begin{array}{l}\text { da di, dang } \\
\text { mu a xi }\end{array}$ & $\begin{array}{l}\text { QTP-EBT- } \\
0047\end{array}$ & Tree & MCB & 0.85 & Other: timber (stems). \\
\hline Plantaginaceae & Plantago depressa Willd. & jia gu ma & $\begin{array}{l}\text { QTP-EBT- } \\
0011\end{array}$ & Herb & $\mathrm{Fi}$ & 0.54 & $\begin{array}{l}\text { Food: cooked vegetable, used to make soup } \\
\text { (whole plants). }\end{array}$ \\
\hline Polygonaceae & $\begin{array}{l}\text { Polygonum lapathifolium L. } \\
\text { var. salicifolium Sibth. }\end{array}$ & liang luo & $\begin{array}{l}\text { QTP-EBT- } \\
0243\end{array}$ & Herb & ASM & 0.37 & Other: tobacco substitute (seeds, flowers). \\
\hline Polygonaceae & $\begin{array}{l}\text { Polygonum macrophyllum D. } \\
\text { Don }\end{array}$ & rong bu & $\begin{array}{l}\text { QTP-EBT- } \\
0086\end{array}$ & Herb & ASM & 1.10 & $\begin{array}{l}\text { Food: staple food, a substitute for tsampa } \\
\text { (seeds)Medicinal: decoction, used to treat } \\
\text { arthralgia (roots). }\end{array}$ \\
\hline Polygonaceae & $\begin{array}{l}\text { Polygonum tortuosum D. } \\
\text { Don }\end{array}$ & $\begin{array}{l}\text { bi yong } \\
\text { jiong, yang } \\
\text { lou }\end{array}$ & $\begin{array}{l}\text { QTP-EBT- } \\
0088\end{array}$ & Herb & ASM & 0.41 & Other: tobacco substitute (seeds, flowers). \\
\hline Polygonaceae & $\begin{array}{l}\text { Rheum acuminatum Hook. f. } \\
\text { \& Thoms. ex Hook. }\end{array}$ & jing la & $\begin{array}{l}\text { QTP-EBT- } \\
0230\end{array}$ & Herb & MCB & 0.34 & $\begin{array}{l}\text { Food: cooked vegetable, to be eaten directly after } \\
\text { peeling (stems). }\end{array}$ \\
\hline Polygonaceae & Rumex nepalensis Spreng. & $\begin{array}{l}\text { jiong xiong, } \\
\text { ye ke ya ma }\end{array}$ & $\begin{array}{l}\text { QTP-EBT- } \\
0091\end{array}$ & Herb & ASM & 1.73 & $\begin{array}{l}\text { Food: vegetable, the stems eaten directly after } \\
\text { peeling and the flowers eaten directly (stems, } \\
\text { flowers).Medicinal: raw, used to relieve altitude } \\
\text { sickness and to treat dyspepsia (leaves). }\end{array}$ \\
\hline Polypodiaceae & $\begin{array}{l}\text { Polypodiodes subamoena (C. } \\
\text { B. Clarke) Ching }\end{array}$ & $\begin{array}{l}\text { ai dei bu de, } \\
\text { en ni }\end{array}$ & $\begin{array}{l}\text { QTP-EBT- } \\
0043\end{array}$ & Herb & MCB & 0.47 & $\begin{array}{l}\text { Medicinal: poultice of stem water extracts, used to } \\
\text { treat chapped skin (stems).Ritual plant: divination } \\
\text { (leaves). }\end{array}$ \\
\hline Pteridiaceae & $\begin{array}{l}\text { Pteridium aquilinum var. } \\
\text { latiusculum (Desv.) Underw. } \\
\text { ex Heller }\end{array}$ & $\begin{array}{l}\text { da po xiu, } \\
\text { da fei, yo }\end{array}$ & $\begin{array}{l}\text { QTP-EBT- } \\
0068\end{array}$ & Herb & MCB & 0.83 & Food: cooked vegetable (stems). \\
\hline
\end{tabular}


Table 2 List of plants and fungi used by the Lhoba people in Douyu Village, Longzi County, Tibet, China (Continued)

\begin{tabular}{|c|c|c|c|c|c|c|c|}
\hline Family name & Scientific name & $\begin{array}{l}\text { Local } \\
\text { name(s) }\end{array}$ & $\begin{array}{l}\text { Voucher } \\
\text { number }\end{array}$ & Habit & Habitat $^{\mathrm{a}}$ & $\mathrm{Cl}$ & Local uses (parts used) \\
\hline Ranunculaceae & $\begin{array}{l}\text { Aconitum kongboense } \\
\text { Lauener }\end{array}$ & wo miu & $\begin{array}{l}\text { QTP-EBT- } \\
0251\end{array}$ & Herb & SBS & 0.07 & $\begin{array}{l}\text { Hunting plant: used to make poisoned arrows for } \\
\text { hunting (root tubers).Medicinal: poisonous plant } \\
\text { (root tuber). }\end{array}$ \\
\hline Ranunculaceae & $\begin{array}{l}\text { Paeonia ludlowii (Stern \& } \\
\text { G.Taylor) D.Y.Hong }\end{array}$ & $\begin{array}{l}\text { ba bu ba xi, } \\
\text { bian ma se } \\
\text { pu (Tibetan) }\end{array}$ & $\begin{array}{l}\text { QTP-EBT- } \\
0028\end{array}$ & Shrub & SBS & 0.80 & $\begin{array}{l}\text { Food: staple food, seed powder used to make } \\
\text { tsampa (seeds); edible oil (seeds).Medicinal: } \\
\text { poultice, used to dye hair (seeds oil). }\end{array}$ \\
\hline Ranunculaceae & Clematis tenuifolia Royle & a shen da li & $\begin{array}{l}\text { QTP-EBT- } \\
0070\end{array}$ & Liana & SBS & 0.22 & $\begin{array}{l}\text { Medical: veterinary, decoction, used to treat } \\
\text { animal skin infection (whole plants); poisonous } \\
\text { plant, leaf epidermis causes human skin allergy } \\
\text { (leaves). }\end{array}$ \\
\hline Ranunculaceae & Thalictrum finetii Boivin & $\begin{array}{l}\text { nie ba } \\
\text { (Tibetan) }\end{array}$ & $\begin{array}{l}\text { QTP-EBT- } \\
0048\end{array}$ & Herb & MCB & 1.07 & $\begin{array}{l}\text { Dye plant (roots).Other: detergent substitute } \\
\text { (roots). }\end{array}$ \\
\hline Rhamnaceae & $\begin{array}{l}\text { Berchemia longipedicellata Y. } \\
\text { L. Chen \& P. K. Chou }\end{array}$ & $\begin{array}{l}\text { guo lang, bu } \\
\text { da xing xi }\end{array}$ & $\begin{array}{l}\text { QTP-EBT- } \\
0006\end{array}$ & Shrub & SBS & 1.80 & $\begin{array}{l}\text { Food: fruit (fruits); beverage, fresh or dry fruits in } \\
\text { boiled water to drink (fruits). }\end{array}$ \\
\hline Rhamnaceae & $\begin{array}{l}\text { Berchemia yunnanensis } \\
\text { Franch. }\end{array}$ & guo lang & $\begin{array}{l}\text { QTP-EBT- } \\
0075\end{array}$ & Shrub & SBS & 1.93 & $\begin{array}{l}\text { Food: fruit (fruits); beverage, fresh or dry fruits in } \\
\text { boiled water to drink (fruits). }\end{array}$ \\
\hline Rhamnaceae & $\begin{array}{l}\text { Rhamnus dumetorum } \\
\text { Schneid }\end{array}$ & $\begin{array}{l}\text { da niu da } \\
\text { wei }\end{array}$ & $\begin{array}{l}\text { QTP-EBT- } \\
0191\end{array}$ & Shrub & SBS & 0.12 & Fodder: yak feed (leaves). \\
\hline Rosaceae & Cerasus rufa Wall. & $\begin{array}{l}\text { ge ga ke, ge } \\
\text { le gong, ge } \\
\text { ga a xi }\end{array}$ & $\begin{array}{l}\text { QTP-EBT- } \\
0076\end{array}$ & Tree & MCB & 1.41 & $\begin{array}{l}\text { Agricultural tool: used to make agricultural tools } \\
\text { (branches).Hunting plant: food for bears (fruits). }\end{array}$ \\
\hline Rosaceae & $\begin{array}{l}\text { Cotoneaster microphyllus } \\
\text { Wall. ex Lindl. var. } \\
\text { thymifolius (Baker) Koehne }\end{array}$ & se ba & $\begin{array}{l}\text { QTP-EBT- } \\
0057\end{array}$ & Shrub & SBS & 0.73 & $\begin{array}{l}\text { Agricultural tool: used to make agricultural tools } \\
\text { (branches). }\end{array}$ \\
\hline Rosaceae & Fragaria gracilis Lozinsk. & jing bu lei & $\begin{array}{l}\text { QTP-EBT- } \\
0226\end{array}$ & Herb & MCB & 1.00 & Food: fruit (fruits). \\
\hline Rosaceae & $\begin{array}{l}\text { Rosa sericea Lindl. f. } \\
\text { glandulosa Yu \& Ku }\end{array}$ & xiu, xiu gu xi & $\begin{array}{l}\text { QTP-EBT- } \\
0003\end{array}$ & Shrub & SBS & 1.78 & $\begin{array}{l}\text { Food: fruit (fruits); beverage, roots soaked in water } \\
\text { (roots).Medicinal: raw, used to treat anemia and to } \\
\text { maintain beauty and youth (fruits). }\end{array}$ \\
\hline Rosaceae & $\begin{array}{l}\text { Rosa webbiana Wall. ex } \\
\text { Royle }\end{array}$ & $\begin{array}{l}\text { da li meng } \\
\text { duo }\end{array}$ & $\begin{array}{l}\text { QTP-EBT- } \\
0077\end{array}$ & Shrub & SBS & 1.37 & $\begin{array}{l}\text { Food: fruit (fruits); vegetable, raw or cooked } \\
\text { (stems). }\end{array}$ \\
\hline Rosaceae & $\begin{array}{l}\text { Rubus biflorus Buch.Ham. ex } \\
\text { Smith }\end{array}$ & $\begin{array}{l}\text { ren bu dao } \\
\text { wei, ri bu }\end{array}$ & $\begin{array}{l}\text { QTP-EBT- } \\
0023\end{array}$ & Shrub & SBS & 1.59 & $\begin{array}{l}\text { Food: fruit (fruits); beverage, fruit in boiled water } \\
\text { to drink (fruits). }\end{array}$ \\
\hline Rosaceae & Rubus ellipticus Smith & zi ga & $\begin{array}{l}\text { QTP-EBT- } \\
0227\end{array}$ & Shrub & SBS & 1.00 & Food: fruit (fruits). \\
\hline Rosaceae & Sorbus rehderiana Koehne & ma mu & $\begin{array}{l}\text { QTP-EBT- } \\
0236\end{array}$ & Tree & MCB & 0.32 & Hunting plant: food for bears (fruits). \\
\hline Saxifragaceae & $\begin{array}{l}\text { Ribes alpestre Wall. ex } \\
\text { Decne. }\end{array}$ & $\begin{array}{l}\text { dong nai ya } \\
\text { yi, jiong duo } \\
\text { (Tibetan) }\end{array}$ & $\begin{array}{l}\text { QTP-EBT- } \\
0022\end{array}$ & Shrub & SBS & 1.39 & Food: fruit (fruits). \\
\hline Saxifragaceae & $\begin{array}{l}\text { Ribes laciniatum Hook. f. \& } \\
\text { Thoms. }\end{array}$ & ku nu & $\begin{array}{l}\text { QTP-EBT- } \\
0246\end{array}$ & Tree & MCB & 1.00 & Food: fruit (fruits). \\
\hline Saxifragaceae & $\begin{array}{l}\text { Saxifraga umbellulata Hook. } \\
\text { f. \& Thoms. }\end{array}$ & $\begin{array}{l}\text { bi xi mi, di } \\
\text { zha (Tibetan) }\end{array}$ & $\begin{array}{l}\text { QTP-EBT- } \\
0020\end{array}$ & Herb & SBS & 0.32 & $\begin{array}{l}\text { Medicinal: decoction, used as choleretic (whole } \\
\text { plants). }\end{array}$ \\
\hline Solanaceae & Anisodus luridus Link \& Otto & ruo ge bu & $\begin{array}{l}\text { QTP-EBT- } \\
0225\end{array}$ & Herb & MCB & 0.41 & $\begin{array}{l}\text { Fodder: yak feed (leaves).Medicinal: poultice, } \\
\text { placed on cuts or broken bones to reduce } \\
\text { swelling and hasten healing (roots). }\end{array}$ \\
\hline Solanaceae & Solanum nigrum $\mathrm{L}$. & he le & $\begin{array}{l}\text { QTP-EBT- } \\
0099\end{array}$ & Herb & $\mathrm{Fi}$ & 0.83 & Food: cooked vegetable (stems and leaves). \\
\hline Tamaricaceae & Myricaria squamosa Desv. & $\begin{array}{l}\text { qu xiu en } \\
\text { guo }\end{array}$ & $\begin{array}{l}\text { QTP-EBT- } \\
0094\end{array}$ & Shrub & ASM & 0.25 & $\begin{array}{l}\text { Medicinal: raw, tender stems and leaves used to } \\
\text { treat colds and used as an antitussive (stems and } \\
\text { leaves). }\end{array}$ \\
\hline Rubiaceae & Leptodermis pilosa Diels & $\begin{array}{l}\text { zha ma li se } \\
\text { (Tibetan) }\end{array}$ & $\begin{array}{l}\text { QTP-EBT- } \\
0060\end{array}$ & Shrub & SBS & 0.73 & Food: nectar used as seasoning (flower). \\
\hline
\end{tabular}


Table 2 List of plants and fungi used by the Lhoba people in Douyu Village, Longzi County, Tibet, China (Continued)

\begin{tabular}{|c|c|c|c|c|c|c|c|}
\hline Family name & Scientific name & $\begin{array}{l}\text { Local } \\
\text { name(s) }\end{array}$ & $\begin{array}{l}\text { Voucher } \\
\text { number }\end{array}$ & Habit & Habitat $^{\mathrm{a}}$ & $\mathrm{Cl}$ & Local uses (parts used) ${ }^{\mathbf{b}}$ \\
\hline Thymelaeaceae & Stellera chamaejasme L. & c & $\begin{array}{l}\text { QTP-EBT- } \\
0039\end{array}$ & Herb & MCB & 0.59 & Other: used to make paper (roots). \\
\hline Thymelaeaceae & $\begin{array}{l}\text { Wikstroemia lungtzeensis S. } \\
\text { C. Huang }\end{array}$ & xiu xin & $\begin{array}{l}\text { QTP-EBT- } \\
0054\end{array}$ & Shrub & SBS & 0.54 & Other: used to make paper (barks). \\
\hline Ulmaceae & Betula utilis D. Don & che ba & $\begin{array}{l}\text { QTP-EBT- } \\
0238\end{array}$ & Tree & MCB & 0.20 & $\begin{array}{l}\text { Artware: used to make wooden bowls and spoons } \\
\text { (stem). }\end{array}$ \\
\hline Umbelliferae & $\begin{array}{l}\text { Heracleum candicans var. } \\
\text { obtusifolium (Wall. ex de } \\
\text { Candolle) F. T. Pu \& M. F. } \\
\text { Watson }\end{array}$ & dong bu & $\begin{array}{l}\text { QTP-EBT- } \\
0042\end{array}$ & Herb & MCB & 1.02 & Food: substitute for staple foods (stems). \\
\hline Umbelliferae & $\begin{array}{l}\text { Pimpinella xizangense Shan } \\
\& \mathrm{Pu}\end{array}$ & mi ru & $\begin{array}{l}\text { QTP-EBT- } \\
0040\end{array}$ & Herb & MCB & 1.80 & $\begin{array}{l}\text { Food: substitute for staple foods (stems); } \\
\text { seasoning (fruits). }\end{array}$ \\
\hline Umbelliferae & Vicatia thibetica de Boiss. & en nin, jia & $\begin{array}{l}\text { QTP-EBT- } \\
0019\end{array}$ & Herb & SBS & 2.15 & $\begin{array}{l}\text { Food: cooked vegetable, used to make soup } \\
\text { (roots); staple food, mixed with flour to make } \\
\text { cakes (leaves).Medicinal: decoction, used to treat } \\
\text { high blood pressure and to relieve altitude } \\
\text { sickness (roots). }\end{array}$ \\
\hline Urticaceae & Urtica dioica $\mathrm{L}$. & $\begin{array}{l}\text { xia di, sha } \\
\text { zhi (Tibetan) }\end{array}$ & $\begin{array}{l}\text { QTP-EBT- } \\
0217\end{array}$ & Herb & SBS & 0.63 & $\begin{array}{l}\text { Fodder: mixed with tsampa to feed yaks } \\
\text { (flowers).Food: cooked vegetable (flowers, leaves). }\end{array}$ \\
\hline Urticaceae & $\begin{array}{l}\text { Urtica membranifolia C. J. } \\
\text { Chen }\end{array}$ & $\begin{array}{l}\text { xia dei, bu } \\
\text { xu bu you }\end{array}$ & $\begin{array}{l}\text { QTP-EBT- } \\
0102\end{array}$ & Herb & $\mathrm{Fi}$ & 0.73 & Food: cooked vegetable (leaves). \\
\hline \multicolumn{8}{|l|}{ Fungi } \\
\hline Agaricaceae & $\begin{array}{l}\text { Leucoagaricus leucothites } \\
\text { (Vittad.) Wasser }\end{array}$ & da ying & $\begin{array}{l}\text { QTP-EBT- } \\
0221\end{array}$ & Herb & SBS & 0.76 & $\begin{array}{l}\text { Food: boiled, barbecued, in fried dishes, or raw } \\
\text { (entire fruit body). }\end{array}$ \\
\hline
\end{tabular}

${ }^{\mathrm{a}} \mathrm{Fi}$, fields; SBS, subalpine broadleaf deciduous shrublands; $M C B$ mixed coniferous and broadleaf forests; ASM alpine shrublands and meadows. ${ }^{\mathrm{b}} \mathrm{Tsampa}$ a traditional staple food of the Lhoba usually made of highland barley. ${ }^{\circ}$ The Lhoba name was forgotten

Chinese Plant Red Book [33], and Paeonia ludlowii (Stern \& G. Taylor) D. Y. Hong was listed on the Threatened Species List of China's Higher Plants [34].

Herbaceous species were the plants most used by the Lhoba people (54 species, 60.0\%), while 22 shrub (24.4\%), 9 tree $(10.0 \%)$, and 5 liana (5.6\%) species were used. In our survey, the most commonly used parts of these plants were fruits (31 species), followed by whole plants (23 species), stems (17 species), leaves (15 species), roots (14 species), flowers (9 species), a combination of stems and leaves (5 species), and bark (3 species).

Figure 3 shows that all habitats, from the field vegetation at the valley bottoms to the alpine shrublands and meadows, are used for plant collection. Of the habitats, the number of species collected was highest in the

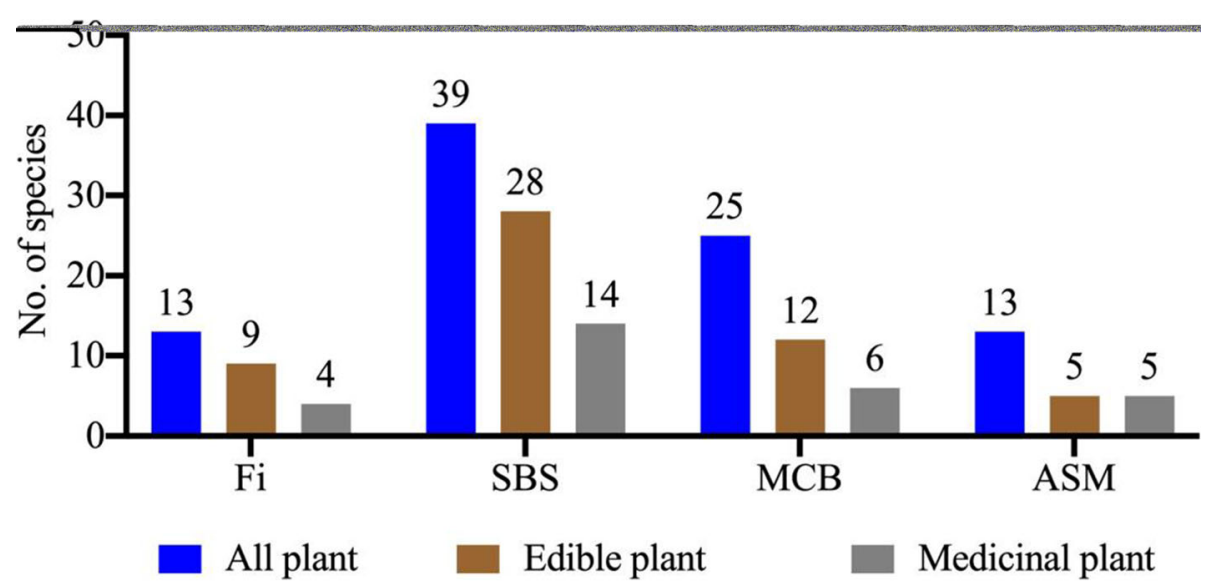

Fig. 3 Habitats of all used plants, edible plants and medicinal plants in the study area. Fi, fields; SBS, subalpine broadleaf deciduous shrublands; $M C B$, mixed coniferous and broadleaf forests; ASM, alpine shrublands and meadows 
subalpine broadleaf deciduous shrubland and lowest in the alpine shrubland and meadow habitat. Moreover, except for in fields, the species richness of all plants, edible plants, and medicinal plants collected in naturally occurring vegetation showed a clear altitudinal trend in Douyu Village, declining from lower altitudes to higher altitudes.

\section{Diversity of wild plant uses}

Our study showed that there are 55 species of edible plants and fungi, 29 species of medicinal plants, and 38 species used in the daily life of the Lhoba people for activities such as hunting (7 species) and for specific uses such as fodder (6 species), rituals (5 species), dyes (4 species), agricultural tools (3 species), detergent substitutes (clothes and hand) (3 species), papermaking (2 species), tobacco substitutes (2 species), processing technology ( 2 species), fuel ( 2 species), timber (1 species), and other uses (1 species) (Fig. 4).

\section{Wild edible plants}

In this study, fifty-four wild plant species were identified as being used as food. According to the eating habits of the Lhoba people, these plants are eaten as vegetables, fruits, staple foods, oil and seasoning, and beverages. Among these edible plants, the most widely used species were vegetables ( 22 species, $28.6 \%$ ), followed by fruits (15 species, $27.1 \%$ ), staple foods (14 species, $17.2 \%$ ), seasoning and oil (10 species, 15.2\%), and beverages (9 species, $12.0 \%$ ). Vegetables are the most commonly used wild edible plants of the Lhoba people, and they play an important role in the lives of the inhabitants of Douyu Village. Vegetables, such as Vicatia thibetica de Boiss., Taraxacum calanthodium Dahlst., T. maurocarpum Dahlst., and Malva verticillata L., are most commonly cooked in water and used to make soup. The Lhoba people still maintain the tradition of eating raw vegetables. For example, stems of Rheum acuminatum Hook. f. \& Thoms. ex Hook. and Rumex nepalensis Spreng. can be eaten directly after being peeled. Fruits are another important food in the daily life of the Lhoba people, accounting for $27.1 \%$ of their diet. Local people eat wild fruits, such as Berchemia yunnanensis Franch., B. longipedicellata, and Hippophae rhamnoides L. subsp. gyantsensis Rousi. Of the edible plants, fruits and vegetables are the foods most widely used by the Lhoba people in Douyu. This result is consistent with the results of several previous studies in the Himalayas in which fruits and vegetables were found to play an important role in the diets of those living in these regions [9-11, 35, 36].

Edible plants are also commonly used for staple foods in Douyu. A total of 14 species were found to be consumed, and these plants are usually eaten during times of food shortage and famine. Traditionally, the rhizomes of Polygonatum verticillatum (L.) All. and the roots of Codonopsis convolvulacea Kurz. var. vinciflora (Kom.) L. T. Shen are used as starchy foods. Currently, seed powders of Paeonia ludlowii, Polygonum macrophyllum D. Don, and Incarvillea beresowskii Batalin are used as tsampa. In particular, P. ludlowii is an endangered species [34] that is restricted to Longzi County and Linzhi County of southeastern Tibet [37]. Recently, this plant has been cultivated by local people in their courtyards for food as well as for ornamental purposes due to its beautiful flowers. P. ludlowii seeds can be ground into powder to make tsampa. In Douyu, $P$. ludlowii seed powder combined with the powder of locally grown black highland barley was used to develop a product, “hei-qing-ke mu-dan fen" (黑青稞牡丹粉) (Fig. 5). Moreover, the Lhoba people in Douyu consume a variety

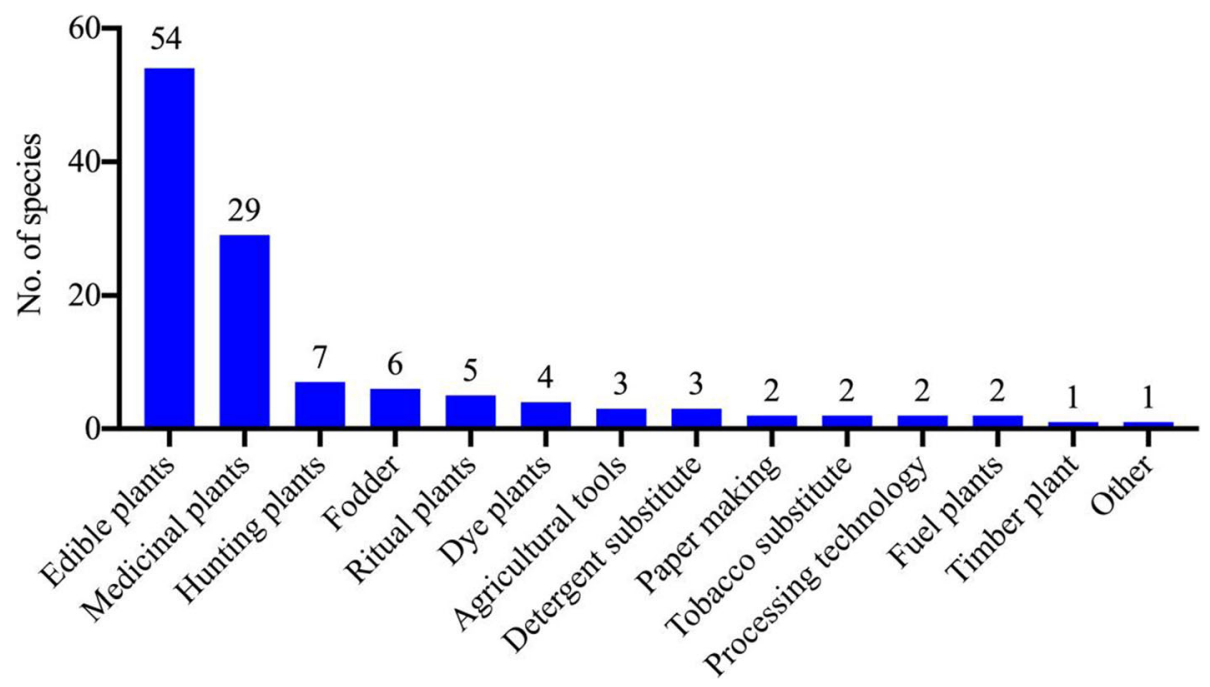

Fig. 4 Plants used for different purposes in the study area 

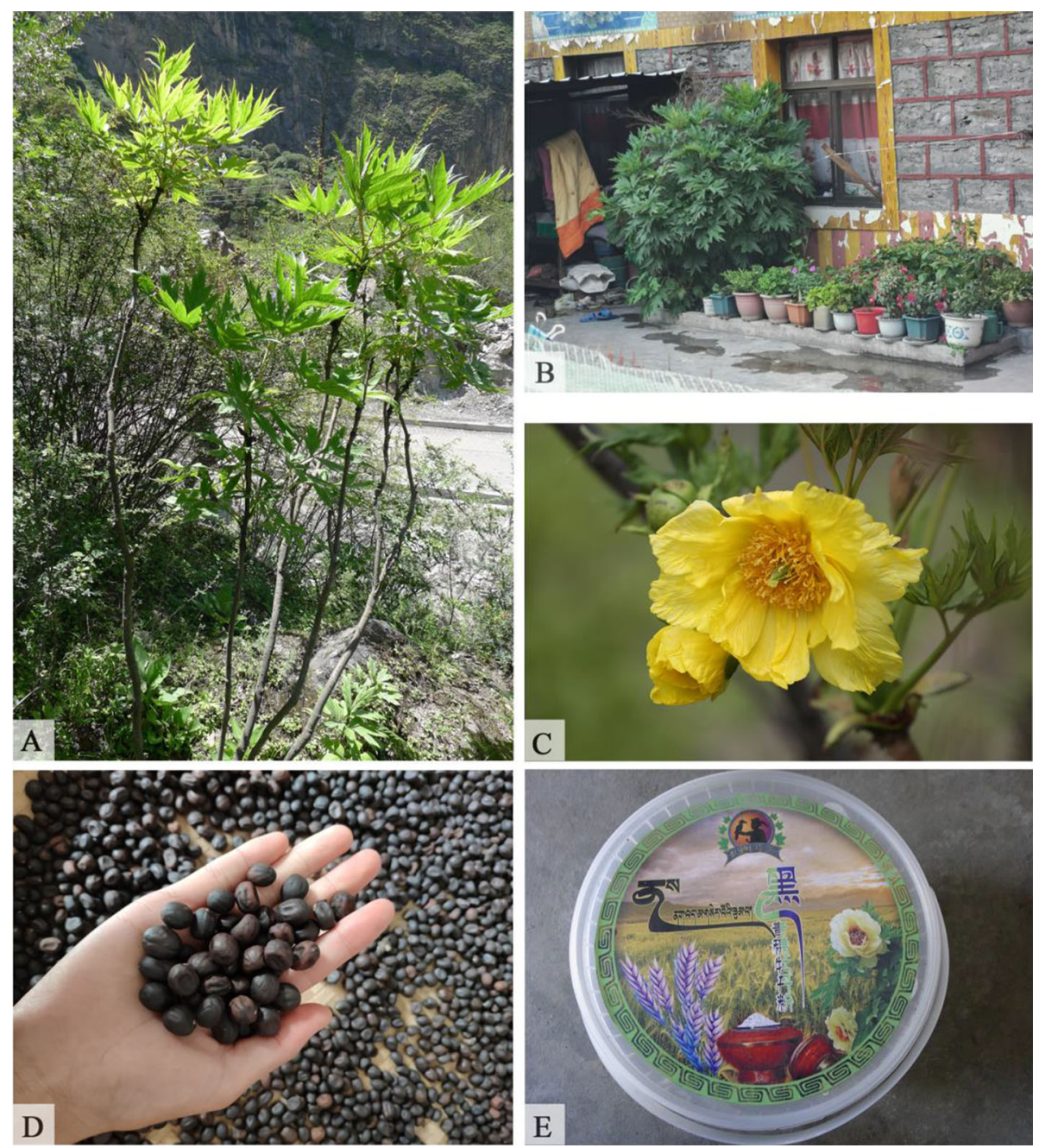

Fig. 5 Paeonia ludlowii, an endangered plant, is used for tsampa, oils, and dying hair. A Habitat of $P$. ludlowii. B Cultivated in a courtyard. C Flower. D Seeds. E The product called "hei-qing-ke mu-dan fen" (黑青稞牡丹粉)

of wild oils and seasoning plants, which are commonly used in daily cuisine to improve a dish's taste. Among these seasoning plants, four species belong to the genus Allium, including A. chrysanthum Regel, A. fasciculatum Rendle (Fig. 6), A. hookeri Thwaites, and A. sikkimense Baker. These wild plants are popular condiments of the local people and serve as seasoning for improving flavor when they are cooked, usually by boiling or frying, with other vegetables or meat. In addition, the Lhoba used nine species for beverages. These plants are used to make tea substitutes or yellow wine or are boiled in hot water and served as traditional beverages.

\section{Wild medicinal plants}

During the investigation, we recorded information on 29 medicinal plants used by the Lhoba people in Douyu Village. The greatest proportion of medicinal plants consisted of herbs $(69.0 \%)$, followed by shrubs $(21.0 \%)$, trees
(7.0\%), and one liana (3.0\%). These results are similar to those of several previous studies in the Himalayas [10, 11, 38]. Parts of these medicinal plants used include whole plants $(37.9 \%)$, followed by fruits $(24.1 \%)$, roots (24.1\%), leaves $(1.0 \%)$, flowers $(1.0 \%)$, bark $(1.0 \%)$, and stems $(1.0 \%)$. There are four main types of prepared traditional medicines: decoctions, medicinal foods, poultices, and medical liquors. Decoctions are the most commonly used type of prepared traditional medicine, with a total of 16 species used in decoctions, and medicinal foods are the second most often consumed type of prepared traditional medicine (11 species), consumed directly or after cooking. Four species are used in poultices, either mashed into a paste or made into a solid extract. Only one species is used for the preparation of medical liquor. A decoction being the most commonly used type of prepared traditional medicine is consistent with the results of other studies [39-41]. 

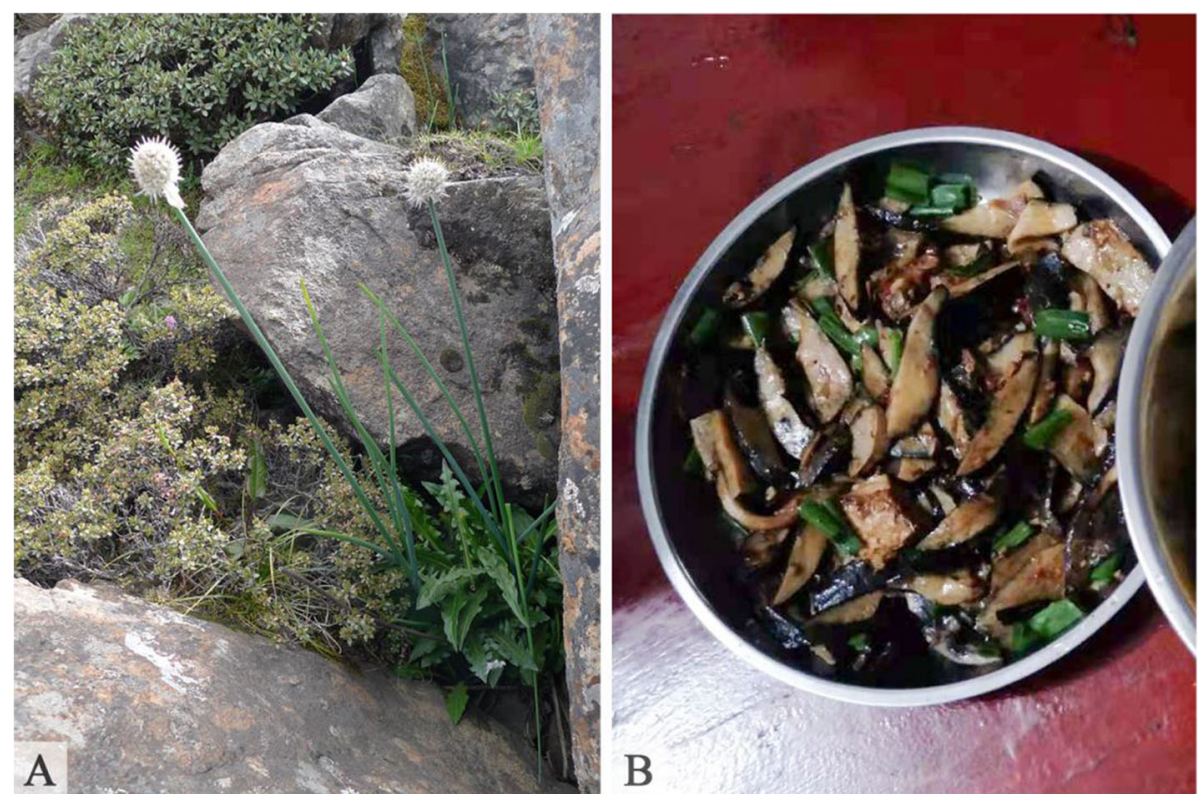

Fig. 6 Allium fasciculatum leaves serve as seasoning in local dishes. A A. fasciculatum habitat. B Stir-fried with other vegetables

According to the information provided by the informants, all illnesses reported to be treated by the wild medicinal plants are classified into 11 categories in Table 3. The ICF results ranged from 0 to 0.96 for the 11 illness categories, with the highest ICF values for diseases of blood forming organs $(\mathrm{ICF}=0.96)$ and gastrointestinal diseases $(\mathrm{ICF}=0.95)$, followed by the ICF values for diseases in animals (0.94) and urology (0.94)-related diseases. Living in remote regions and eating too varied of a diet or excessively, especially in high-altitude areas, may lead to gastrointestinal diseases [42, 43]. Similarly, our study shows that many medicinal plants are commonly used to treat gastrointestinal disease. For example, Berberis kongboensis and B. xanthophloea are used to treat diarrhea, while Rumex nepalensis is used for indigestion by the Lhoba people. Treating diseases of blood-forming organs is also one of the most common uses of medicinal plants, such as Sinopodophyllum hexandrum, Rosa sericea f. glandulosa, and Sambucus adnata Wall.

\section{Wild plants used for other purposes}

In addition to edible and medicinal plants, we also recorded 38 plant species used in the daily life of the Lhoba, including those for hunting, fodder, and ritual uses (Fig. 4). After edible plants and medicinal plants, use for hunting (7 species) was the third most important

Table. 3 Ethnobotanical consensus index for traditional medicinal plant use categories

\begin{tabular}{|c|c|c|c|c|}
\hline Use category & Terms & $\begin{array}{l}\text { Number of } \\
\text { taxa }\left(N_{\mathrm{t}}\right)\end{array}$ & $\begin{array}{l}\text { Number of use } \\
\text { reports }\left(N_{\mathrm{ur}}\right)\end{array}$ & $\begin{array}{l}\text { Informant consensus } \\
\text { factor (ICF) }\end{array}$ \\
\hline Cancer & Oncological diseases & 1 & 1 & - \\
\hline Circulatory system & $\begin{array}{l}\text { Altitude sickness, high blood pressure, high-altitude } \\
\text { pulmonary edema }\end{array}$ & 8 & 89 & 0.92 \\
\hline Cosmetics & Maintain beauty, youth, and dark hair & 3 & 17 & 0.88 \\
\hline Dermatologic disorders & Chapped skin, scalds, wounds & 3 & 22 & 0.90 \\
\hline $\begin{array}{l}\text { Diseases of blood- } \\
\text { forming organs }\end{array}$ & Anemia & 3 & 50 & 0.96 \\
\hline $\begin{array}{l}\text { Gastrointestinal } \\
\text { problems }\end{array}$ & Diarrhea, dyspepsia, stomachache, choleretic issues & 4 & 60 & 0.95 \\
\hline Poisons & Poisons, insecticides & 5 & 16 & 0.73 \\
\hline Respiratory complaints & Colds, sore throat, coughs & 3 & 26 & 0.92 \\
\hline Skeletomuscular system & Arthralgia, lumbago, fractures & 5 & 49 & 0.92 \\
\hline Urology & Gynecological diseases, nephropathy & 4 & 54 & 0.94 \\
\hline Veterinary & Skin infections, ectoparasitic control, oxytocic & 3 & 37 & 0.94 \\
\hline
\end{tabular}


category. Traditionally, hunting is a primary aspect of the lifestyle of the Lhoba people used to maintain their livelihoods [17]. It is therefore not surprising that the local population uses a variety of plants for hunting. For instance, a poisonous plant, Aconitum kongboense Lauener, is used to produce poison arrows for hunting, while Rhodiola bupleuroides (Wall. ex Hook. f. \& Thoms.) S. H. Fu, R. quadrifida (Pall.) Fisch. \& Mey., Sinocrassula densirosulata (Praeger) A. Berger, and Sorbus rehderiana Koehne are used as bait to hunt animals. Six species, the whole plants of Polygonatum verticillatum, Medicago sativa L., and Vicia cracca L.; the leaves of Rhamnus dumetorum Schneid and Anisodus luridus Link \& Otto; and the flowers of Urtica dioica L. are used as fodder for grazing animals.

Ritual use of plants plays a particularly important role in the daily life of Douyu Village. The Lhoba people use these plants to please the deities and ask for their support for human health and wellbeing. Most ritual plants commonly used in religious activities of the Lhoba people, such as Rhododendron anthopogon D. Don, $R$. lepidotum Wall. ex G. Don, and Sabina convallium (Rehd. \& Wils.) Cheng \& W. T. Wang, are burned as incense to please the deities. The custom of using incense in Douyu is similar to that of the Lhoba ethnic group in Milin County [11] and Medog County [12] in China, and this custom is derived from the religious rituals of Tibetan Buddhism. The fresh or dry branches of these species are burned in a censer, which is placed on the flat roof of a house or at the entrance to the village. The first two plants are collected on high-elevation mountains while herding yaks and are occasionally used as incense. The latter species, however, is collected in the subalpine broadleaf deciduous shrub vegetation surrounding the village and is usually burned as incense during the daily morning ritual. Quercus aquifolioides Rehd. \& Wils. is another type of ritual plant and is used for decoration in sacrificial ceremonies. The use of this species is related to a Lhoba festival traditionally held in Douyu in autumn to celebrate harvest [19]. At present, the festival is called the Yu Luo Cultural Festival, is the most important religious festival of the Lhoba people in Douyu, and is usually held at the end of July every year. Before the festival, local people go to the mountains and collect ge rui xi (Q. aquifolioides). A sacred yak is made from this plant (apart from the head, for which a real yak head is used), which is placed in the middle of a square during the sacrificial ceremony. On that day, Lhoba people dress in traditional costumes, surround the sacred yak and dance and sing songs. Under the guidance of the wizard, local people celebrated harvest this year and prayed for a good harvest and greater wellbeing during the coming year. Certainly, the occasion provided a feast with abundant food after the sacrificial ceremony. When local people were asked why the sacred yak was made from Quercus aquifolioides, they said that this plant was one of a few broadleaf evergreen species surrounding Douyu Village. They also use Polypodiodes subamoena (C. B. Clarke) Ching for simple divination activities to determine the appropriateness of an action. When local people go out hunting or collecting, they use the leaves of this plant to predict probable outcomes. In this activity, a leaflet of the lamina of the plant is selected to denote good luck, and then leaflets are alternately designated good or bad; favorable outcomes for the day's work are expected if the terminal leaflet is good.

\section{Mushrooms}

Our study only recorded one fungal species used by the Lhoba people in Douyu Village. It is worth noting that the mushroom Leucoagaricus leucothites (Vittad.) Wasser was encountered during transect walks for supplementary field surveys in August 2020. Although our objectives were to focus on vascular plants, we also added this species to our dataset because the local leader and translator mentioned that a variety of mushrooms were distributed in Douyu Village and were commonly eaten by local people. In our survey, the entire fruiting body of $L$. leucothites was usually eaten boiled, barbecued, in a fried dish, or raw (Table 2).

\section{Importance of plant use}

Based on the CI values, the most important plants in this study area were Berberis xanthophloea $(\mathrm{CI}=2.37)$, B. kongboensis $(\mathrm{CI}=2.37)$, Sinopodophyllum hexandrum $(\mathrm{CI}=2.24)$, Vicatia thibetica $(\mathrm{CI}=2.15)$, and Hippophae rhamnoides subsp. gyantsensis $(\mathrm{CI}=2.02)$ (Table 2).

These species are used for more than one purpose. For instance, the fresh fruits of Berberis kongboensis and $B$. xanthophloea are edible without any particular preparation, their flowers can be used to make a salad, and the bark, phloem, fruits, and flowers can be used to treat diarrhea. In the same way, the ripe fruits of Sinopodophyllum hexandrum are edible, and its fruits can be used as a treatment for human anemia, a cosmetic to maintain beauty and youth, and an oxytocic for yaks. Roots of Vicatia thibetica are used to treat high blood pressure and relieve altitude sickness, its roots are also used as vegetables to stew with meat or to cook in soup, and its leaves can be mixed with flour and baked into cakes. Fruits of Hippophae rhamnoides subsp. gyantsensis are used to relieve altitude sickness, its fresh fruits are edible, and its fresh or dried fruits can be soaked in wine or water to be used as a beverage. 
Comparison of utilized plant species among Lhoba ethnic groups in different areas

To investigate how environmental factors may have affected plant use by the Lhoba people, the recorded data were subjected to regional comparisons among the Lhoba ethnic groups in three counties (Longzi County, Medog County, and Milin County) in China. There is only one plant, namely, Pteridium aquilinum var. latiusculum (Desv.) Underw. ex Heller (Fig. 7), used by the Lhoba people in all three areas. There are eight plant species, Aconitum kongboense, Berberis kongboensis, Berchemia yunnanensis, Polygonum tortuosum D. Don, Pteridium aquilinum var. latiusculum, Quercus aquifolioides, Rubus biflorus Buch.-Ham. ex Smith, and Sinopodophyllum hexandrum, used by Lhoba ethnic groups in both Longzi County and Milin County. Pteridium aquilinum var. latiusculum and Solanum nigrum L. are used in both Longzi County and Medog County (Fig. 7). Similarly, there were only two species, Pteridium aquilinum var. latiusculum and Senecio scandens Buch.-Ham. ex D. Don, used in both Milin County and Medog County [9].

\section{Discussion}

Useful wild plants along an altitudinal gradient

Our study showed that all habitats, from the field vegetation at the valley bottoms to the alpine shrublands and meadows, are used for plant collection. The species richness of all used plants, edible plants, and medicinal plants collected in each vegetation type (apart from field vegetation) decreased with increasing altitude (Fig. 3).
These results are consistent with those from previous studies [20, 25]. For example, according to Salick et al., the number of species used by the Dusun people of Mt. Kinablu is significantly and negatively correlated with altitude [20]. Similarly, Weckerle et al. observed that collection sites for useful plants in the Hengduan Mountains substantially increase in number with decreasing altitude [25]. In addition, a similar pattern occurs in different places in the Qinling Mountains between Shaanxi and Gansu provinces, where the number of wild edible plants used has been found to be highest near Xi'an [44], and then west from there on the edges of the Tibetan Plateau, studies have found fewer edible species at intermediate elevations [45], with the lowest number at higher elevations [46].

Previous studies have shown that convenience or accessibility for collecting a plant contributes to the tendency for local people to collect that plant in the vicinity of settlements [20, 25, 47-49]. Our study area, Douyu Village, contains complex terrain in a small geographical area with an extreme variety in altitude. Thus, which wild plants are used by the Lhoba people in Douyu is strongly influenced by the accessibility of plant collection sites. Our study shows that $58.2 \%$ of the total plant species used are collected in the vicinity of the village, where the habitats are field vegetation and subalpine broadleaf deciduous shrubland (Fig. 3).

On the other hand, cultural value is another factor influencing local people to collect plants, such as ritual plants, medicinal plants, and plants with special uses, at

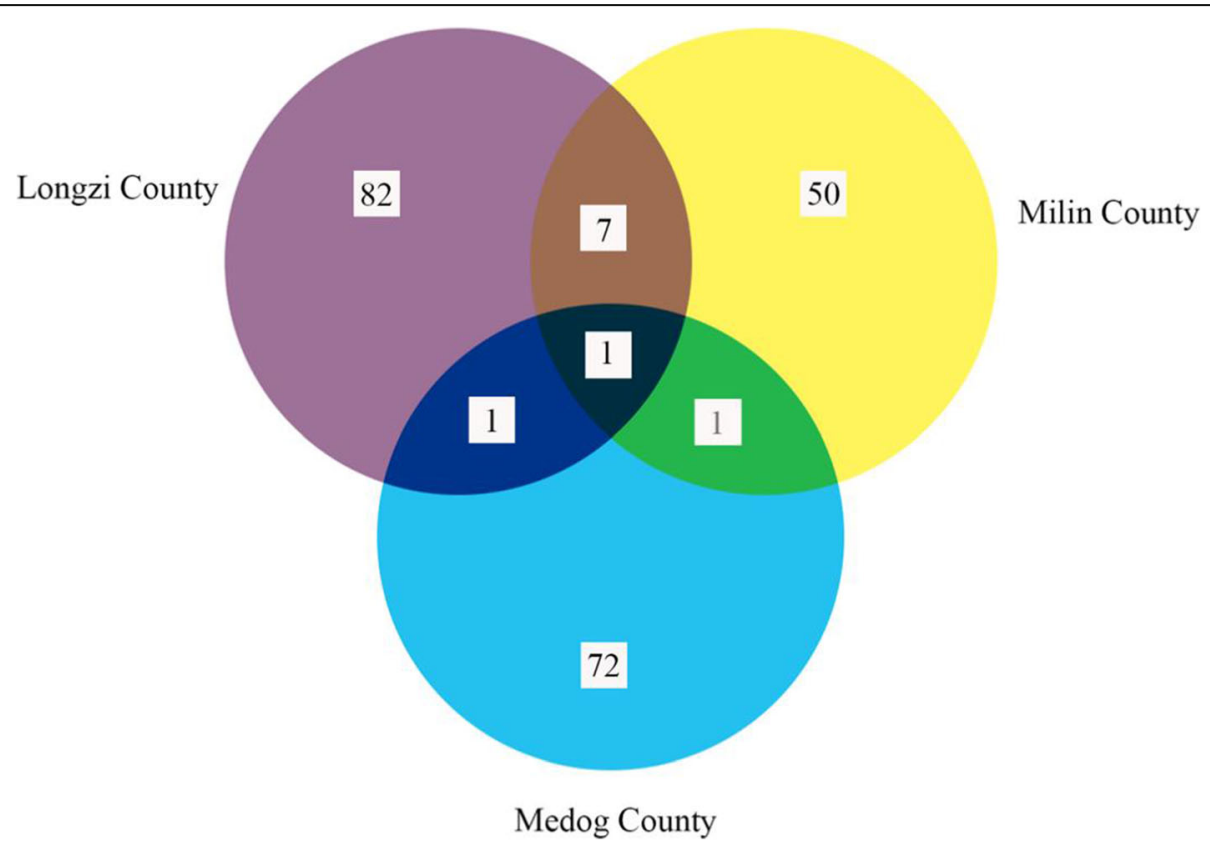

Fig. 7 Comparison of plants used in the three counties. Notes: purple, Douyu Village (Longzi County); yellow, Nanyi Township (Milin County); blue, Damu Township (Medog County) 
remote locations and in high-altitude places. The Lhoba people's faith in the original religion of "all things and spirits" involves praying to the gods and seeking blessings $[17,19,29]$. Our results showed that ritual plants play an important role in daily life in Douyu, with a total of five species used in ritual activities. The habitats where ritual plants are collected are not near the village but at relatively remote distances. For instance, local people climb high-elevation mountains to collect Rhododendron anthopogon and R. lepidotum in alpine shrublands and meadows. These two plants are burned as incense to please the deities. They also travel a certain geographical distance to collect Quercus aquifolioides in mixed coniferous and broadleaf forests. This plant is used for decoration at sacrificial ceremonies.

Similarly, the Lhoba people collect important or particular plants at remote and high altitudes. For example, Berberis kongboensis, Sinopodophyllum hexandrum, and Hippophae rhamnoides subsp. gyantsensis are collected in mixed coniferous and broadleaf forests. Swertia hookeri C. B. Clarke is collected in alpine shrub areas and meadows. Its roots are used to treat traumatic injury, wounds, and high blood pressure. The Lhoba people also collect Polygonum lapathifolium L. var. salicifolium Sibth. and P. tortuosum from alpine shrublands and meadow habitats to serve as tobacco substitutes.

Therefore, our study demonstrates that accessibility and cultural value affect where useful plants are collected.

\section{Important plant uses}

Our study showed that some plants are important based on their CI values (Table 2). These plants exhibit a wide range of uses and are highly recognized and accepted locally.

Berberis kongboensis $(\mathrm{CI}=2.37)$ and $B$. xanthophloea $(\mathrm{CI}=2.37)$ are the most important plants in Douyu Village. These two species, which are distributed in southeastern Tibet, are endemic to Tibet [32]. B. kongboensis and $B$. xanthophloea are used in almost the same manner by the Lhoba people. Their fresh fruits are eaten directly; their flowers are used to make salad; their bark, phloem, fruits, flowers are used to treat diarrhea; and their fruits are also used in dyes. The uses of fresh fruit are similar to those for other Berberis species in the Himalayas [11, 15, 50, 51]. This genus is also rich in pharmacologically active compounds, such as berberine, berbamine, palmatine, oxyacanthine, and jatrorrhizine [52]. Berberine is widely used to treat diarrhea by inhibiting intestinal inflammation and regulating intestinal bacteria [53-57].

Sinopodophyllum hexandrum $(\mathrm{CI}=2.24)$, the only species of this genus in China, is an endangered species native to the Himalayan region [32, 33]. In our survey, its ripe fruits were eaten directly, and its fruits were also renowned for their medicinal value in Douyu Village. Local people use it as a treatment for anemia, a cosmetic to maintain beauty and youth, or as an oxytocic for yaks. A previous study observed that this plant is a popular fruit [11, 15], and it is called Himalayan mayapple [58]. This species exhibits effective anticancer activity. Its secondary metabolite is effective against tumors, gynecological diseases, and rheumatism [59]. In Tibetan medicine, it is used to promote blood circulation and eliminate blood stasis and to treat gynecological diseases, bruises, dermatological diseases, and antenatal pain [60].

Vicatia thibetica $(\mathrm{CI}=2.15)$ is a perennial herb distributed in the Himalayan and Hengduan Mountains [32]. In Douyu, its leaves are mixed with flour and baked into cakes, and the roots are used as a vegetable to stew with meat or cook in soup. Moreover, the roots can also be used to treat high blood pressure and relieve altitude sickness. Acute and subacute toxicity studies on root and leaf extracts of $V$. thibetica have shown that there is no toxic effect after long-term use, and it is quite safe as food [61, 62].

Hippophae rhamnoides subsp. gyantsensis $(\mathrm{CI}=2.02)$ is distributed in the southeastern Himalayas [32]. In our survey, it was locally used as a fruit, a beverage, and a treatment for altitude sickness. Fruits of this plant contain fat-soluble vitamins, fatty acids, carotenoids, and amino acids that are beneficial to health [63-65]. Its fresh fruits are also used in many areas, and berries can be made into jams, juices, and other products $[15,58$, $65,66]$. One new usage was recorded in this study: a medical liquor preparation of its fruits can be used to relieve altitude sickness.

\section{Relationships between utilized wild plants and environment \\ Relationships between utilized wild plants and geographic environments}

In this study, the wild plants used by the Lhoba people in Douyu Village were richly diverse, reflecting the number of species and diversified functions. A total of 91 wild plant and fungal species are used in the daily lives of the Lhoba people for various purposes, serving as edible plants, medicinal plants, hunting aids, fodder, ritual plants, dyes, agricultural tools, detergent substitutes, papermaking materials, and tobacco substitutes (Fig. 4). This result is consistent with previous results from the Eastern Himalayas, which have shown that wild plants provide the sustenance for these communities and form an integral part of the culture and traditions of these communities [9-12].

In our study area, Douyu Village is situated among high mountains and valleys. This village is isolated from other regions in China due to its unique geographic 
environment and difficult road conditions. In these extreme areas, the Lhoba people have thus learned to make full use of the wild plant resources in the area surrounding their village to support their daily needs [17], and this scenario has contributed to the high diversity of traditional knowledge on wild plants. In addition, their cultivated land is quite limited, and agricultural yields are low because of the complex terrain and high altitude. The livelihood of the Lhoba people is dependent on forests and other natural resources apart from agricultural and animal production. It is noted that 14 wild plants are used as staple foods, which may help the local people address seasonal food shortages and withstand famine. For example, the seed powders of Paeonia ludlowii, Polygonum macrophyllum, and Incarvillea beresowskii are used to make tsampa, while rhizomes of Polygonatum verticillatum and the roots of Codonopsis convolvulacea var. vinciflora are used as starchy food.

Moreover, the Lhoba people have accumulated a wealth of knowledge about the traditional uses of wild plants because they have lived in the Eastern Himalayas for hundreds of years. They have developed many unique cultural traditions around useful wild plants. In addition to the edible and medicinal plants, there are diverse wild plant species used in the daily life of the Lhoba documented in this survey. For instance, they use Aconitum kongboense, a poisonous plant, for hunting and Polygonatum verticillatum, Medicago sativa, and Vicia cracca as fodder; Thalictrum finetii Boivin and Impatiens cristata Wall. are used to produce dyes; Cerasus rufa Wall. and Cotoneaster microphyllus Wall. ex Lindl. var. thymifolius (Baker) Koehne are used to make agricultural tools; Wikstroemia lungtzeensis and Stellera chamaejasme are used to make paper. Previous studies have noted that geographical isolation could contribute to the preservation of diverse cultural traditions of local people in Himalayan regions [14] and could help preserve diverse traditional botanical knowledge [9-11, 67]. These findings have shown that useful wild plants are highly diverse in geographically isolated areas, and this diversity in Douyu Village is based not only on the number of species but also on their diversified functions.

\section{Relationships between utilized wild plants and special ecological environments}

Douyu Village is located among high mountains and valleys in the Eastern Himalayas, with temperate and semiarid montane climates. The annual temperature is 4.2 ${ }^{\circ} \mathrm{C}$, and the annual average precipitation is $279.4 \mathrm{~mm}$. This area features very typical vegetation types and species. Along the elevational gradient, vegetation from the low valleys to the high-altitude mountains consists mainly of subalpine broadleaf deciduous shrublands, mixed coniferous and broadleaf forests, and alpine shrublands and meadows.
In particular, the subalpine broadleaf deciduous shrubland is a special vegetation type that is found only in southeastern Tibet. This vegetation can withstand cold and dry climates and poor soils, forming forests on the upper slopes and valleys at altitudes ranging from 3000 to $4000 \mathrm{~m}$ in the Eastern Himalayas. In addition, the vegetation exhibits low coverage, ranging from 20 to $60 \%$, and the dominant plant is Leptodermis pilosa Diels [28]. The Lhoba people make full use of the wild plants in this area. Our study showed that the numbers of all used plants, edible plants, and medicinal plants collected from the subalpine broadleaf deciduous shrub areas were 40 species, 29 species, and 14 species, respectively, and each use category exhibited a high number of species for all vegetation types (Fig. 3). For instance, the uppermost shrub layer primarily includes L. pilosa, Berberis kongboensis, B. xanthophloea, Berchemia longipedicellata, Rhamnus dumetorum, Sabina convallium and Wikstroemia lungtzeensis; the second layer mainly includes Cotoneaster microphyllus var. thymifolius, Dracocephalum tanguticum Maxim., Hedysarum longigynophorum, and Nepeta angustifolia C. Y. Wu; and the herbaceous layer chiefly includes Allium chrysanthum Regel, Arisaema flavum (Forsk.) Schott, Dracocephalum tanguticum, Nepeta angustifolia, Polygonatum verticillatum, Salvia przewalskii Maxim., Sinocrassula densirosulata, and Vicatia thibetica. The lianas include Codonopsis convolvulacea var. vinciflora, Cynanchum saccatum W. T. Wang ex Tsiang \& P. T. Li, and C. auriculatum Royle ex Wight. In addition, some of these listed plants are especially useful, such as Wikstroemia lungtzeensis, a species endemic to Longzi County; the narrow-range species Berberis xanthophloea, Berchemia longipedicellata, Hedysarum longigynophorum, Nepeta angustifolia, and Rosa sericea f. glandulosa; and the endangered species Paeonia ludlowii.

The indigenous practices of the Lhoba people have been affected by a combination of their long history, unique ecological factors involving cold and dry climates, and specific plant resources around their village. Several studies have shown that lifestyles and cultures are significantly influenced by extreme ecological conditions [9, 14, 27, 68, 69]. Thus, different ecological environments result in the presence of different useful plants. Our research showed that the plants useful to the Lhoba people in Longzi County, Milin County, and Medog County in China show substantial differences (Fig. 7). For instance, there is only one plant, Pteridium aquilinum var. latiusculum, used by the Lhoba people in all three counties. Eight of the same species are present in both Longzi and Minlin Counties, two plants occur in both Longzi and Medog Counties, and two species occur in Minlin and Medog Counties (Fig. 7).

Longzi County (Douyu Village), Milin County (Nanyi Township), and Medog County (Damu Township) are 
all located in the eastern Himalayas, and these three counties are relatively isolated from other regions in China. However, the ecological environments differ significantly among these three areas in terms of climate and vegetation types. For example, Douyu experiences a semiarid climate, where the annual average precipitation is only $279.4 \mathrm{~mm}$, and the vegetation is mainly alpinesubalpine broadleaf deciduous shrubland. In contrast, Nanyi Township features temperate to cool temperate climates, the annual average precipitation is $600 \mathrm{~mm}$, and the main vegetation is coniferous broadleaf mixed forests [11]. Damu Township exhibits a subtropical climate, the average annual precipitation is $2400 \mathrm{~mm}$, and the vegetation mainly consists of broadleaf evergreen forests [12]. Compared with that of Damu, the ecologic environments of both Douyu and Nanyi are similar to a certain extent. As a result, there were more plant species shared between Douyu and Nanyi than between Douyu and Damu: the former two shared eight of the same plant species, and the latter two had two of the same plant species. A similar result was also found for Nanyi and Damu. There were only two species shared by Nanyi and Damu due to their relatively different ecological environments (Fig. 7).

Therefore, the extreme environmental conditions of Douyu Village may have affected the lifestyles and cultures of the Lhoba people, leading to substantial plant utilization.

\section{The relevance of this study for rural development, food sovereignty, and food security in the study area}

Local people not only use wild plants to meet their own needs but can also profit from using plant products. Paeonia ludlowii has been developed as a special food product in Douyu Village (Fig. 5). Other plants, such as Sinopodophyllum hexandrum and Hippophae rhamnoides subsp. gyantsensis with high CI values, as well as Pimpinella xizangense and Wikstroemia lungtzeensis that are endemic to Longzi County, also hold potential for rural economic development. To utilize these plants in sustainable ways, it is important to carry out intentional planting in the future.

The opinion of food sovereignty has been raised by the International Peasants' Movement (La Via Campesina, LVC) [70]. Some of the views expressed by LVC are helpful for rural sustainable development. For example, LVC has stated: "food is a key part of culture, and the neoliberal agenda is destroying the very basis of our lives and cultures. We do not accept the hunger and displacement. We demand food sovereignty, which means the right to produce our own food" [71]. The loss of traditional knowledge in rural communities is actually a key problem. This is the reason why we do ethnobotanical studies. We want to try to delay the process of loss.
Although these wild edible plants might be beneficial to the local people, we should also notice the possible hazards of these plants to the human body after consuming them. According to previous reports, humans and animals are affected by Pteridium aquilinum var. latiusculum, which contains ptaquiloside, a carcinogenic toxin [72]. The toxicity of Solanum nigrum is mainly due to the presence of solanine, with symptoms of poisoning in humans including nausea, vomiting, diarrhea, headache, dizziness, loss of speech, fever, sweating, tachycardia, pupil dilation, blindness, mental confusion, convulsions, coma, and death [73]. It is necessary to inform the local people and local government in Longzi County about the hazards of these plants. In two other studies, no acute or subacute toxicity was observed in mice administered Hippophae rhamnoides or Vicatia thibetica [61,74]. However, no toxicological information was found for other wild plants eaten by the Lhoba people in Douyu Village. Researchers should devote more attention to food security.

\section{Conclusion}

This is the first study of wild plants used by the Lhoba people in Douyu Village, Longzi County, in the Eastern Himalayas, and 91 plant and fungal species (43 genera and 39 families) used in their daily life were recorded. The results of this study showed that the species richness of all used plants, edible plants, and medicinal plants collected from different vegetation types (apart from field vegetation) decreased with increasing altitude, and this pattern was mainly influenced by the accessibility of sites and cultural values of plants. Our study demonstrates that the diversity of wild plants used by the Lhoba people is reflected not only in the number of species but also in the diversified functions of wild plants, including edible plants, medicinal plants, plants used for hunting, fodder, ritual plants, and plants used for dyes. On the one hand, Douyu Village is isolated from other regions of China. The Lhoba people have learned to fully use their wild plant resources to support their daily needs, preserve the diverse cultural traditions of Lhoba people and certainly form rich traditional botanical knowledge. On the other hand, the extreme ecological conditions of Douyu Village may have affected the lifestyles and cultures of the Lhoba people. In comparison to the Lhoba in other areas, the Lhoba people in Douyu Village use wild plants extensively. Therefore, the extreme climatic, geographical, and ecological conditions of Douyu Village in the high mountains and valleys contribute to the rich diversity of wild plants used by the Lhoba people.

\section{Acknowledgements}

We are grateful to the local government worker Ms. Zha Xi Yang Jin, the local translator Ms. Ba Sang La Mu, and the local guides Mr. Da Wa, Mr. Zha 
Xi Ci Ren, and Mr. Suo Lang Ba Zhu. We are very grateful to the informants for sharing their knowledge with us. We also thank all of the local people who helped us with the fieldwork. We thank Ms. Yu Zhang at Kunming Institute of Botany, Chinese Academy of Sciences, for help with the field investigation and informant interviews. We also thank Dr. Xiu-Li Zeng at the Institute of Vegetables, Tibet Academy of Agricultural and Animal Husbandry Sciences, for help taking photographs of Paeonia ludlowii flowers.

\section{Authors' contributions}

Yu-Hua Wang and Yue-Hu Wang organized the study team and provided technical support and guidance. CWY and YT designed and executed the research plan. CWY, YT, and YJ wrote the manuscript, which was revised by Yue-Hu Wang. QZC and DXY recorded and organized the data. CWY and YT identified the specimens and reviewed the information. All authors were involved in the drafting and revision of the manuscript and approved the final revision.

\section{Funding}

The study was funded by the "Second Tibetan Plateau Scientific Expedition and Research (No. 2019QZKK0502)" Project of China and the Strategic Priority Research Program of the Chinese Academy of Sciences (No. XDA19050301 and XDA19050303).

\section{Availability of data and materials}

All data generated or analyzed during this study are included in this published article.

\section{Declarations}

Ethics approval and consent to participate

Not applicable.

\section{Consent for publication}

Not applicable.

\section{Competing interests}

The authors declare that they have no competing interests.

\section{Author details}

'Key Laboratory of Economic Plants and Biotechnology and the Yunnan Key Laboratory for Wild Plant Resources, Kunming Institute of Botany, Chinese Academy of Sciences, Kunming 650201, People's Republic of China.

${ }^{2}$ University of Chinese Academy of Sciences, Beijing 100049, China.

Received: 23 March 2021 Accepted: 14 July 2021

Published online: 23 July 2021

\section{References}

1. Mosbrugger V, Favre A, Muellner-Riehl AN, Päckert M, Mulch A. Cenozoic evolution of geobiodiversity in the Tibeto-Himalayan region. In: Hoorn C, Perrigo A, Antonelli A, editors. Mountains, climate and biodiversity. Oxford: Wiley Blackwell; 2018. p. 429-48. https://www.wiley.com/en-gb/Mounta ins\%2C+Climate+and+Biodiversity-p-9781119159889.

2. Myers $\mathrm{N}$, Mittermeier RA, Mittermeier CG, Da Fonseca GAB, Kent J. Biodiversity hotspots for conservation priorities. Nature. 2000,403,853-858. https://doi.org/https://doi.org/10.1038/35002501, 6772.

3. Barthlott W, Mutke J, Rafigpoor D, Kier G, Kreft H. Global centers of vascular plant diversity. Nova Acta Leopoldina. 2005,92,61-83. https://www.resea rchgate.net/publication/230787324_Global_Centers_of_Vascular_Plant_ Diversity.

4. Ding W-N, Ree RH, Spicer RA, Xing Y-W. Ancient orogenic and monsoondriven assembly of the world's richest temperate alpine flora. Science. 2020,369,578-581. https://doi.org/https://doi.org/10.1126/science.abb4484, 6503.

5. Kohler T, Maselli D. Mountains and climate change. From Understanding to Action. Geographica Bernensia, Bern, Switzerland. 2009, https://lib.icimod. org/record/14409/files/5504.pdf.

6. Turin M. A multitude of mountain voices. Sustain Mountain Dev. 2007;52: 11-3 https://lib.icimod.org/record/26331.

7. Gorenflo LJ, Romaine S, Mittermeier RA, Walker-Painemilla K. Co-occurrence of linguistic and biological diversity in biodiversity hotspots and high biodiversity wilderness areas. Proc Natl Acad Sci USA. 2012,109,8032-8037. https://doi.org/https://doi.org/10.1073/pnas.1117511109, 21.

8. Salick J, Staver B, Hart R. Indigenous knowledge and dynamics among Himalayan peoples, vegetation, and climate change. In: Welch-Devine M, Sourdril A, Burke B, editors. Changing climate, changing worlds. Ethnobiology. Cham, Switzerland: Springer; 2020. p. 55-69. https://doi.org/ https://doi.org/10.1007/978-3-030-37312-2_4.

9. Yang J, Chen W-Y, Fu Y, Yang T, Luo X-D, Wang Y-H, Wang Y-H. Medicinal and edible plants used by the Lhoba people in Medog County, Tibet, China. J Ethnopharmacol. 2020,249,112430. https://doi.org/https://doi.org/10.1016/j. jep.2019.112430

10. Li S, Zhang Y, Guo Y, Yang L, Wang Y. Monpa, memory, and change: an ethnobotanical study of plant use in Medog County, South-east Tibet, China. J Ethnobiol Ethnomed. 2020,16,5. https://doi.org/https://doi.org/10.11 86/s13002-020-0355-7, 1.

11. Li F, Zhuo J, Liu B, Jarvis D, Long C. Ethnobotanical study on wild plants used by Lhoba people in Milin County, Tibet. J Ethnobiol Ethnomed. 2015,11,23. https://doi.org/https://doi.org/10.1186/s13002-015-0009-3, 1.

12. Chen $\mathrm{W}-\mathrm{Y}$, Wang $\mathrm{Y}-\mathrm{H}$, Wang $\mathrm{Y}-\mathrm{H}$. The plants traditionally used by the Lhopa people in Medog County, Tibet. Kunming: Yunnan Science and Technology Press; 2019.

13. Rana D, Bhatt A, Lal B. Ethnobotanical knowledge among the semi-pastoral Gujjar tribe in the high altitude (Adhwari's) of Churah subdivision, district Chamba, Western Himalaya. J Ethnobiol Ethnomed. 2019,15,10. https://doi. org/https://doi.org/10.1186/s13002-019-0286-3, 1.

14. Farooquee NA, Saxena KG. Conservation and utilization of medicinal plants in high hills of the central Himalayas. Environ Conserv. 1996,23,75-80. https://doi.org/https://doi.org/10.1017/s0376892900038273, 1.

15. Boesi A. Traditional knowledge of wild food plants in a few Tibetan communities. J Ethnobiol Ethnomed. 2014,10,75. https://doi.org/https://doi. org/10.1186/1746-4269-10-75, 1.

16. Chen L-M, Li J-P. Lhoba: A series of approaching Chinese minorities. Shengyang: Liaoning Nationalities Press; 2015.

17. Editorial Group of Social and Historical Survey Data Series of Tibet. Social history investigation of the Lhoba nationality. Beijing: The Ethnic Publishing House; 2009.

18. Wang M-L. Research review on the Lhoba nationality's intangible cultural heritage. J. Harbin Univ. 2014;35:114-7 http://www.cqvip.com/QK/83151A/2 01412/663431840.html.

19. Editorial Group of Brief History of the Lhoba People. Brief history of the Lhoba people. Beijing: The Ethnic Publishing House; 2009.

20. Salick J, Biun A, Martin G, Apin L, Beaman R. Whence useful plants? A direct relationship between biodiversity and useful plants among the Dusun of Mt. Kinabalu. Biodivers Conserv. 1999,8,797-818. https://doi.org/https://doi. org/10.1023/A:1008853413930, 6.

21. Kala CP. Status and conservation of rare and endangered medicinal plants in the Indian trans-Himalaya. Biol Conserv. 2000,93,371-379. https://doi.org/ https://doi.org/10.1016/S0006-3207(99)00128-7, 3.

22. Kunwar RM, Chaudhary RP. Status, vegetation composition and biomass of forests of Arun valley. East Nepal. Banko Janakari. 2004,14,13-18. https://doi. org/https://doi.org/10.3126/banko.v14i1.17054, 1.

23. Kunwar RM, Bussmann RW. Ethnobotany in the Nepal Himalaya. J Ethnobiol Ethnomed. 2008,4,24. https://doi.org/https://doi.org/10.1186/1746-4269-4-24, 1.

24. Bhattarai KR, Ghimire M. Commercially important medicinal and aromatic plants of Nepal and their distribution pattern and conservation measure along the elevation gradient of the Himalayas. Banko Janakari. 2006,16,3-13. https://doi.org/https://doi.org/10.3126/banko.v16i1.357, 1.

25. Weckerle CS, Huber FK, Yang Y, Sun W. Plant knowledge of the Shuhi in the Hengduan Mountains, Southwest China. Econ Bot. 2006,60,3-23. https://doi. org/https://doi.org/10.1663/0013-0001(2006)60[3:PKOTSI]2.0.CO;2.

26. Zurick D, Pacheco J, Shrestha B, Bajracharya B. Illustrated Atlas of the Himalaya. University of Kentucky Press: Lexington; 2006. https://www.jstor. org/stable/j.ctt2jcm1s

27. Aziz MA, Khan $\mathrm{AH}$, Pieroni A. Ethnoveterinary plants of Pakistan: a review. J Ethnobiol Ethnomed. 2020,16,25. https:/doi.org/10.1186/s13002-020-00369-1, 1.

28. Integrated Scientific Survey Team of Qinghai-Tibet Plateau, Chinese Academy of Sciences. Vegetation in Tibet. Beijing: Science Press; 1988.

29. Yu N-C. Obsessed faith and osessed art: The primitive religion and culture of the Lhoba people. China Tibetol. 1989:145-60 http://qikan.cqvip.com/Qika n/Article/Detail?id=1002909820\&from=Qikan_Search_Index. 
30. Trotter RT, Logan MH. Informant consensus: a new approach for identifying potentially effective medicinal plants. In: Etkin NL, editor. Plants in indigenous medicine and diet: Biobehavioral approaches. Now York: Redgrave Bedford Hills; 1986. p. 91-112.

31. Tardio J, Pardo-de-Santayana M. Cultural importance indices: a comparative analysis based on the useful wild plants of southern Cantabria (northern Spain). Econ Bot. 2008,62,24-39. https://doi.org/https://doi.org/10.1007/ s12231-007-9004-5, 1.

32. Wu Z, Raven PH, Hong D. Flora of China. Beijing: Science Press. St. Louis: Missouri Botanical Garden Press; 1994.

33. Fu L-G. Chinese plant red book. Beijing: Science Press; 1991.

34. Qin H-N, Yang Y, Dong S-Y, He Q, Jia Y, Zhao L-N, Yu S-X, Liu H-Y, Liu B, Yan Y-H, Xiang J-Y, Xia N-H, Peng H, Li Z-Y, Zhang Z-X, He X-J, Yin L-K, Lin Y-L, Liu Q-R, Hou Y-T, Liu Y, Liu Q-X, Cao W, Li J-Q, Chen S-L, Jin X-H, Gao T-G, Chen W-L, Ma H-Y, Geng Y-Y, Jin X-F, Chang C-Y, Jiang H, Cai L, Zang C-X, Wu J-Y, Ye J-F, Lai Y-J, Liu B, Lin Q-W, Xue N-X. Threatened species list of China's higher plants. Biodivers Sci. 2017,25,696-744. https://doi.org/https:// doi.org/10.17520/biods.2017144, 7.

35. Uprety Y, Poudel RC, Shrestha KK, Rajbhandary S, Tiwari NN, Shrestha UB, Asselin $\mathrm{H}$. Diversity of use and local knowledge of wild edible plant resources in Nepal. J Ethnobiol Ethnomed. 2012,8,16. https://doi.org/https:// doi.org/10.1186/1746-4269-8-16, 1.

36. Sundriyal M, Sundriyal RC. Wild edible plants of the Sikkim Himalaya: Nutritive values of selected species. Econ Bot. 2001,55,377-390. https://doi org/https://doi.org/10.1007/BF02866561, 3

37. Hong D-Y, Zhou S, He X, Yuan J, Zhang Y, Cheng F, Zeng X, Wang Y, Zhang $X$. Current status of wild tree peony species with special reference to conservation. Biodivers Sci. 2017,25,781-793. https://doi.org/https://doi.org/1 0.17520/biods.2017129, 7

38. Rokaya MB, Münzbergová Z, Timsina B. Ethnobotanical study of medicinal plants from the Humla district of western Nepal. J. Ethnopharmacol. 2010,130,485-504. https://doi.org/https://doi.org/10.1016/j.jep.2010.05.036, 3.

39. Hu R, Lin C, Xu W, Liu Y, Long C. Ethnobotanical study on medicinal plants used by Mulam people in Guangxi, China. J Ethnobiol Ethnomed. 2020,16,40. https://doi.org/https://doi.org/10.1186/s13002-020-00387-z, 1.

40. Phumthum M, Srithi K, Inta A, Junsongduang A, Tangjitman K, Pongamornkul W, Trisonthi C, Balslev H. Ethnomedicinal plant diversity in Thailand. J Ethnopharmacol. 2018,214,90-98. https://doi.org/https://doi.org/1 0.1016/j.jep.2017.12.003.

41. Scherrer AM, Motti R, Weckerle CS. Traditional plant use in the areas of Monte Vesole and Ascea, Cilento National Park (Campania, Southern Italy). J Ethnopharmacol. 2005,97,129-143. https://doi.org/https://doi.org/10.1016/j. jep.2004.11.002, 1

42. Patterson K, Clark S, Berrang-Ford L, Lwasa S, Namanya D, Twebaze F, Harper SL, IHACC Research Team, Harper SL. Acute gastrointestinal illness in an African Indigenous population: the lived experience of Uganda's Batwa. Rural Remote Health. 2020,20,5141. https://doi.org/https://doi.org/10.22605/ RRH5141.

43. Tariq A, Mussarat S, Adnan M, Abd Allah EF, Hashem A, Alqarawi AA, Ullah R. Ethnomedicinal evaluation of medicinal plants used against gastrointestinal complaints. Biomed Res Int. 2015,2015,892947. https://doi. org/https://doi.org/10.1155/2015/892947, 892914.

44. Kang Y, Luczaj L, Ye S, Zhang S, Kang J. Wild food plants and wild edible fungi of Heihe valley (Qinling Mountains, Shaanxi, central China): herbophilia and indifference to fruits and mushrooms. Acta Soc Bot Pol. 2012,81,405-413. https://doi.org/https://doi.org/10.5586/asbp.2012.044, 4

45. Kang Y, Łuczaj $Ł$, Kang J, Wang F, Hou J, Guo Q. Wild food plants used by the Tibetans of Gongba Valley (Zhouqu county, Gansu, China). J Ethnobiol Ethnomed. 2014,10,20. https://doi.org/https://doi.org/10.1186/1746-4269-1 $0-20,1$.

46. Kang J, Kang Y, Ji X, Guo Q, Jacques G, Pietras M, Łuczaj N, Li D, Łuczaj Ł. Wild food plants and fungi used in the mycophilous Tibetan community of Zhagana (Tewo County, Gansu, China). J Ethnobiol Ethnomed. 2016,12,21. https://doi.org/https://doi.org/10.1186/s13002-016-0094-y, 1.

47. Kunwar RM, Fadiman M, Hindle T, Suwal MK, Adhikari YP, Baral K, Bussmann R. Composition of forests and vegetation in the Kailash Sacred Landscape, Nepal. J Forestry Res. 2020,31,1625-1635. https://doi.org/https://doi.org/10.1 007/s11676-019-00987-w, 5

48. Kunwar RM, Baral K, Paudel P, Acharya RP, Thapa-Magar KB, Cameron M, Bussmann RW. Land-use and socioeconomic change, medicinal plant selection and biodiversity resilience in far western Nepal. Plos One. 2016,11,e167812. https://doi.org/https://doi.org/10.1371/journal.pone.01 $67812,12$.

49. Kunwar RM, Shrestha K, Malla S, Acharya T, Sementelli AJ, Kutal D, Bussmann RW. Relation of medicinal plants, their use patterns and availability in the lower Kailash Sacred Landscape, Nepal. Ethnobot Res Appl. 2019,18,1-14. https://doi.org/https://doi.org/10.17348/era.18.7.1-14.

50. Wang J, Seyler BC, Ticktin T, Zeng Y, Ayu K. An ethnobotanical survey of wild edible plants used by the Yi people of Liangshan Prefecture, Sichuan Province, China. J Ethnobiol Ethnomed. 2020,16,10. https://doi.org/https:// doi.org/10.1186/s13002-019-0349-5, 1.

51. Singh A, Nautiyal MC, Kunwar RM, Bussmann RW. Ethnomedicinal plants used by local inhabitants of Jakholi block, Rudraprayag district, western Himalaya, India. J Ethnobiol Ethnomed. 2017,13,49. https://doi.org/https:// doi.org/10.1186/s13002-017-0178-3, 1.

52. Abdykerimova S, Sakipova Z, Nakonieczna S, Koch W, Biernasiuk A, Grabarska A, Malm A, Kozhanova K, Kukula-Koch W. Superior antioxidant capacity of Berberis iliensis-HPLC-Q-TOF-MS based phytochemical studies and spectrophotometric determinations. Antioxidants. 2020,9,504. https:// doi.org/https://doi.org/10.3390/antiox9060504, 6 .

53. Li L, Cui H, Li T, Qi J, Chen H, Gao F, Tian X, Mu Y, He R, Lv S, Chu F, Xu B, Wang $P$, Lei $H$, Xu H, Wang $C$. Synergistic effect of berberine-based Chinese medicine assembled nanostructures on diarrhea-predominant irritable bowel syndrome in vivo. Front Pharmacol. 2020,11,1210. https://doi.org/ https://doi.org/10.3389/fphar.2020.01210.

54. Di Pierro F, Bertuccioli A, Giuberti R, Saponara M, Ivaldi L. Role of a berberinebased nutritional supplement in reducing diarrhea in subjects with functional gastrointestinal disorders. Minerva Gastroenterol. Dietol. 2020,66,29-34. https:// doi.org/https://doi.org/10.23736/S1121-421X.19.02649-7, 1.

55. Yue S-J, Liu J, Wang W-X, Wang A-T, Yang X-Y, Guan H-S, Wang C-Y, Yan D. Berberine treatment-emergent mild diarrhea associated with gut microbiota dysbiosis. Biomed Pharmacother. 2019,116,109002. https://doi.org/https:// doi.org/10.1016/j.biopha.2019.109002

56. Imenshahidi $\mathrm{M}, \mathrm{Hosseinzadeh} \mathrm{H}$. Berberine and barberry (Berberis vulgaris): a clinical review. Phytother Res. 2019,33,504-523. https://doi.org/https://doi. org/10.1002/ptr.6252, 3.

57. Hou Q, Zhu S, Zhang C, Huang Y, Guo Y, Li P, Chen X, Wen Y, Han Q, Liu F. Berberine improves intestinal epithelial tight junctions by upregulating A20 expression in IBS-D mice. Biomed Pharmacother. 2019,118,109206. https:// doi.org/https://doi.org/10.1016/j.biopha.2019.109206.

58. Ju Y, Zhuo J, Liu B, Long C. Eating from the wild: diversity of wild edible plants used by Tibetans in Shangri-la region, Yunnan, China, J Ethnobiol Ethnomed. 2013,9,28. https://doi.org/https://doi.org/10.1186/1746-4269-9-28, 1.

59. Wang Q-H, Guo S, Yang X-Y, Zhang Y-F, Shang M-Y, Shang Y-H, Xiao J-J, Cai S-Q. Flavonoids isolated from Sinopodophylli Fructus and their bioactivities against human breast cancer cells. Chin J Nat Med. 2017,15,225-233. https://doi.org/https://doi.org/10.1016/S1875-5364(17)30039-0, 3.

60. Yang J-S, Ge Sang Suo Lang XJ-C, Yang Q-S, Wang X-H. China Tibetan medicinal plant correction. Kunming: Yunnan Science and Technology Press; 2017.

61. Zhan D, Wei R-R, Ma Q-G, He J-W, Duo Jie Jia, Ren Zeng Ping Cuo, Se Zhen. Acute and subacute toxicity study of extracts from roots and leaves of Vicatia thibetica de Boiss. Nat Prod Res Dev. 2020,32,1228-34,1267. https:// doi.org/https://doi.org/10.16333/j.1001-6880.2020.7.019.

62. Zhang W-M, Duan Z-H, Sun F, Rao G-X. The chemical constituents from the roots of Vicatia thibetica. Nat Prod Res Dev. 2004,16,218-219. https://doi.org/ https://doi.org/10.16333/j.1001-6880.2004.03.010.

63. Tkacz K, Wojdylo A, Turkiewicz IP, Ferreres F, Moreno DA, Nowicka P. UPLCPDA-Q/TOF-MS profiling of phenolic and carotenoid compounds and their influence on anticholinergic potential for AChE and BuChE inhibition and on-line antioxidant activity of selected Hippophae rhamnoides L. cultivars. Food Chemistry. 2020,309,125766. https://doi.org/https://doi.org/10.1016/j. foodchem.2019.125766.

64. Sytařová I, Orsavová J, Snopek L, Mlček J, Byczyński Ł, Mišurcová L. Impact of phenolic compounds and vitamins $C$ and $E$ on antioxidant activity of sea buckthorn (Hippophae rhamnoides L.) berries and leaves of diverse ripening times. Food Chem. 2020,310,125784. https://doi.org/https://doi.org/10.1016/ j.foodchem.2019.125784.

65. Ivanišová E, Blašková M, Terentjeva M, Grygorieva O, Vergun O, Brindza J, Kačániová M. Biological properties of sea buckthorn (Hippophae rhamnoides L.) derived products. Acta Sci Pol Technol Aliment. 2020,19,195-205. https:// doi.org/https://doi.org/10.17306/J.AFS.2020.0809. 
66. Guo R, Chang X, Guo X, Brennan CS, Li T, Fu X, Liu RH. Phenolic compounds, antioxidant activity, antiproliferative activity and bioaccessibility of Sea buckthorn (Hippophae rhamnoides L.) berries as affected by in vitro digestion. Food Funct. 2017,8,4229-4240. https://doi.org/https://doi.org/10.1 039/c7fo00917h, 11.

67. Zhang Y, Yang L-X, Li M-X, Guo Y-J, Li S, Wang Y-H. The best choices: the diversity and functions of the plants in the home gardens of the Tsang-la (Motuo Menba) communities in Yarlung Tsangpo Grand Canyon, Southwest China. J Ethnobiol Ethnomed. 2020,16,50. https://doi.org/https://doi.org/1 0.1186/s13002-020-00395-z, 1.

68. Pant SD. The social economy of the Himalaya. London: George Allen and Unwin; 1935. p. 264.

69. Bhandari JS. Structure and change among the borderland communities of Kumaon Himalaya. In: Lall JS, Moddie AD, editors. The Himalaya: aspects of change. New Delhi: Oxford University Press; 1981. p. 204-16.

70. La Via Campesina. Towards Farmers' Right. 2020. https://viacampesina.org/ en/towards-farmers-rights14/. Accessed 12 June 2021.

71. La Via Campesina. Bangalore Declaration of The Via Campesina, Bangalore. 2020. https://viacampesina.org/en/bangalore-declaration-of-the-via-ca mpesina/. Accessed 12 June 2021.

72. Gil da Costa RM, Bastos MMSM, Oliveira PA, Lopes C. Bracken-associated human and animal health hazards: chemical, biological and pathological evidence. J Hazard Mater. 2012,203-4,1-12. https://doi.org/https://doi.org/1 0.1016/j.jhazmat.2011.12.046.

73. Jain R, Sharma A, Gupta S, Sarethy IP, Gabrani R. Solanum nigrum: current perspectives on therapeutic properties. Altern Med Rev. 2011;16(1):78-85. https://pubmed.ncbi.nlm.nih.gov/21438649/.

74. Ali R, Ali R, Jaimini A, Nishad DK, Mittal G, Chaurasia OP, Kumar R, Bhatnagar A, Singh SB. Acute and sub acute toxicity and efficacy studies of Hippophae rhamnoides based herbal antioxidant supplement. Indian J Pharm.

2012,44,504-508. https://doi.org/https://doi.org/10.4103/0253-7613.99329, 4.

\section{Publisher's Note}

Springer Nature remains neutral with regard to jurisdictional claims in published maps and institutional affiliations.

Ready to submit your research? Choose BMC and benefit from:

- fast, convenient online submission

- thorough peer review by experienced researchers in your field

- rapid publication on acceptance

- support for research data, including large and complex data types

- gold Open Access which fosters wider collaboration and increased citations

- maximum visibility for your research: over $100 \mathrm{M}$ website views per year

At $\mathrm{BMC}$, research is always in progress.

Learn more biomedcentral.com/submissions 